TTP99-49

December 1999

\title{
Differential Equations for Two-Loop Four-Point Functions
}

\author{
T. Gehrmann and E. Remiddi \\ Institut für Theoretische Teilchenphysik, Universität Karlsruhe, D-76128 Karlsruhe, Germany
}

\begin{abstract}
At variance with fully inclusive quantities, which have been computed already at the two- or three-loop level, most exclusive observables are still known only at one-loop, as further progress was hampered so far by the greater computational problems encountered in the study of multi-leg amplitudes beyond one loop. We show in this paper how the use of tools already employed in inclusive calculations can be suitably extended to the computation of loop integrals appearing in the virtual corrections to exclusive observables, namely two-loop four-point functions with massless propagators and up to one off-shell leg. We find that multi-leg integrals, in addition to integration-by-parts identities, obey also identities resulting from Lorentz-invariance. The combined set of these identities can be used to reduce the large number of integrals appearing in an actual calculation to a small number of master integrals. We then write down explicitly the differential equations in the external invariants fulfilled by these master integrals, and point out that the equations can be used as an efficient method of evaluating the master integrals themselves. We outline strategies for the solution of the differential equations, and demonstrate the application of the method on several examples.
\end{abstract}

\footnotetext{
*Supported by Alexander-von-Humboldt Stiftung, permanent address: Dipartimento di Fisica, Università di Bologna, I-40126 Bologna, Italy
} 


\section{Introduction}

Perturbative corrections to many inclusive quantities have been computed to the two- and three-loop level in past years. From the technical point of view, these inclusive calculations correspond to the computation of multi-loop two-point functions, for which many elaborate calculational tools have been developed. In contrast, corrections to exclusive observables, such as jet production rates, could up to now only be computed at the one-loop level. These calculations require the computation of multi-leg amplitudes to the required number of loops, which beyond the one-loop level turn out to be a calculational challenge obstructing further progress. Despite considerable progress made in recent times, many of the two-loop integrals relevant for the calculation of jet observables beyond next-to-leading order are still unknown. One particular class of yet unknown integrals appearing in the two-loop corrections to three jet production in electron-positron collisions, to two-plus-one jet production in electron-proton collisions and to vector boson plus jet production in proton-proton collisions are two-loop four-point functions with massless internal propagators and one external leg off-shell.

Taking two-loop four-point integrals arising in the calculation of Feynman diagrams in covariant gauges (non-covariant gauges introduce integrals of a structure beyond the treatment of this paper) as an example, we elaborate on in this paper several techniques to compute multi-leg amplitudes beyond one loop. We demonstrate how integration-by-parts identities (already known to be a very valuable tool in inclusive calculations) and identities following from Lorentz-invariance (which are non-trivial only for integrals depending on at least two independent external momenta) can be used to reduce the large number of different integrals appearing in an actual calculation to a small number of master integrals. This reduction can be carried out mechanically (by means of a small chain of computer programs), without explicit reference to the actual structure of the integrals under consideration and can also be used for the reduction of tensor integrals beyond one loop.

The master integrals themselves, however, can not be computed from these identities. We derive differential equations in the external momenta for them. Solving these differential equations, it is possible to compute the master integrals without explicitly carrying out any loop integration, so that this technique appears to be a valuable alternative to conventional approaches for the computation of multi-loop integrals.

The plan of the paper is as follows. In Section 2 we review the derivation of the integration by parts (IBP) identities and introduce the Lorentz invariance (LI) identities. In Section 3 the differential equations for the master amplitudes are obtained. The practical application of these tools is outlined in detail in Section 4 on a self-contained rederivation of the one-loop massless box integral with one off-shell leg. Section 5 contains examples of massless two-loop four-point functions with one off-shell leg, evaluated for arbitrary space-time dimension. We show which of these functions can be reduced to simpler functions and which are genuine master integrals, and compute some of the master integrals by solving the corresponding differential equations. Finally, Section 6 contains conclusions and an outlook on potential future applications of the tools developed here. The higher transcendental functions appearing in our results for the one-loop and two-loop integrals are summarised in an Appendix, where we also discuss how these functions can be expanded around the physical number of space-time dimensions.

\section{Reduction to Master Integrals}

Any scalar massless two-loop integral can be brought into the form

$$
I\left(p_{1}, \ldots, p_{n}\right)=\int \frac{\mathrm{d}^{d} k}{(2 \pi)^{d}} \frac{\mathrm{d}^{d} l}{(2 \pi)^{d}} \frac{1}{D_{1}^{m_{1}} \ldots D_{t}^{m_{t}}} S_{1}^{n_{1}} \ldots S_{q}^{n_{q}},
$$

where the $D_{i}$ are massless scalar propagators, depending on $k, l$ and the external momenta $p_{1}, \ldots, p_{n}$ while $S_{i}$ are scalar products of a loop momentum with an external momentum or of the two loop momenta. The topology (interconnection of propagators and external momenta) of the integral is uniquely determined by specifying the set $\left(D_{1}, \ldots, D_{t}\right)$ of $t$ different propagators in the graph. The integral itself is then specified by the powers $m_{i}$ of all propagators and by the selection $\left(S_{1}, \ldots, S_{q}\right)$ of scalar products and their powers 
$\left(n_{1}, \ldots, n_{q}\right)$. (all the $m_{i}$ are positive integers greater or equal to 1 , while the $n_{i}$ are greater or equal to 0$)$. Integrals of the same topology with the same dimension $r=\sum_{i} m_{i}$ of the denominator and same total number $s=\sum_{i} n_{i}$ of scalar products are denoted as a class of integrals $I_{t, r, s}$. The integration measure and scalar products appearing the above expression are in Minkowskian space, with the usual causal prescription for all propagators. The loop integrations are carried out for arbitrary space-time dimension $d$, which acts as a regulator for divergencies appearing due to the ultraviolet or infrared behaviour of the integrand (dimensional regularisation, 1, 2).

Any four-point function depends on three linearly independent external momenta, $p_{1}, p_{2}$ and $p_{3}$. At the two-loop level, one can combine the two loop momenta $k$ and $l$ and these external momenta to form 9 different scalar products involving $k$ or $l$. As the propagators present in the graph are (linearly independent) combinations of scalar products, only $9-t$ different scalar products can appear explicitly in an integral with $t$ different propagators. Since a two-loop four-point function can have at most seven different propagators, as can be found by considering the insertion of a propagator into a one-loop four-point function, one has in general $t \leq 7$, while the minimum number of massless propagators in a two-loop graph is $t=3$, corresponding to a two-point function. The number of different two-loop four-point integrals for given $t$ (number of different propagators), $r$ (sum of powers of all propagators) and $s$ (sum of powers of all scalar products) can be computed from simple combinatorics:

$$
N\left(I_{t, r, s}\right)=\left(\begin{array}{c}
r-1 \\
r-t
\end{array}\right)\left(\begin{array}{c}
8-t+s \\
s
\end{array}\right) .
$$

The number $N\left(I_{t, r, s}\right)$ of the integrals grows quickly as $r, s$ increase, but the integrals are related among themselves by various identities. One class of identities follows from the fact that the integral over the total derivative with respect to any loop momentum vanishes in dimensional regularisation

$$
\int \frac{\mathrm{d}^{d} k}{(2 \pi)^{d}} \frac{\partial}{\partial k^{\mu}} J(k, \ldots)=0,
$$

where $J$ is any combination of propagators, scalar products and loop momentum vectors. $J$ can be a vector or tensor of any rank. The resulting identities [2, 3] are called integration-by-parts (IBP) identities and can for two-loop integrals be cast into the form

$$
\begin{gathered}
\int \frac{\mathrm{d}^{d} k}{(2 \pi)^{d}} \frac{\mathrm{d}^{d} l}{(2 \pi)^{d}} \frac{\partial}{\partial k^{\mu}} v^{\mu} f\left(k, l, p_{i}\right)=0, \\
\int \frac{\mathrm{d}^{d} k}{(2 \pi)^{d}} \frac{\mathrm{d}^{d} l}{(2 \pi)^{d}} \frac{\partial}{\partial l^{\mu}} v^{\mu} f\left(k, l, p_{i}\right)=0,
\end{gathered}
$$

where the integrand $f\left(k, l, p_{i}\right)$ is a scalar function, containing propagators and scalar products and $v_{\mu}$ can be any external or loop momentum vector. As a consequence, one obtains for a graph with $m$ loops and $n$ independent external momenta a total number of $N_{\text {IBP }}=m(n+m)$. For a two-loop four-point function, this results in ten IBP identities for each integrand.

In addition to the IBP identities, one can also exploit the fact that all integrals under consideration are Lorentz scalars (or, perhaps more precisely, "d-rotational" scalars), which are invariant under a Lorentz (or $d$-rotational) transformation of the external momenta. In order to derive the resulting Lorentz invariance (LI) identities, we consider an infinitesimal Lorentz transformation

$$
p^{\mu} \rightarrow p^{\mu}+\delta p^{\mu}=p^{\mu}+\delta \epsilon_{\nu}^{\mu} p^{\nu} \quad \text { with } \quad \delta \epsilon_{\nu}^{\mu}=-\delta \epsilon_{\mu}^{\nu},
$$

which should not change the scalar Feynman integral

$$
I\left(p_{1}+\delta p_{1}, \ldots, p_{n}+\delta p_{n}\right)=I\left(p_{1}, \ldots, p_{n}\right)
$$

Expanding

$$
I\left(p_{1}+\delta p_{1}, \ldots, p_{n}+\delta p_{n}\right)=I\left(p_{1}, \ldots, p_{n}\right)+\delta p_{1}^{\mu} \frac{\partial}{\partial p_{1}^{\mu}} I\left(p_{1}, \ldots, p_{n}\right)+\ldots+\delta p_{n}^{\mu} \frac{\partial}{\partial p_{n}^{\mu}} I\left(p_{1}, \ldots, p_{n}\right)
$$


one arrives at

$$
\delta \epsilon_{\nu}^{\mu}\left(p_{1}^{\nu} \frac{\partial}{\partial p_{1}^{\mu}}+\ldots+p_{n}^{\nu} \frac{\partial}{\partial p_{n}^{\mu}}\right) I\left(p_{1}, \ldots, p_{n}\right)=0 .
$$

Since $\delta \epsilon_{\nu}^{\mu}$ has six independent components, the above equation contains up to six LI identities. These are however not always linearly independent. To determine the maximum number of linearly independent identities, one uses the antisymmetry of $\delta \epsilon_{\nu}^{\mu}$ to obtain

$$
\left(p_{1}^{\nu} \frac{\partial}{\partial p_{1 \mu}}-p_{1}^{\mu} \frac{\partial}{\partial p_{1 \nu}}+\ldots+p_{n}^{\nu} \frac{\partial}{\partial p_{n \mu}}-p_{n}^{\mu} \frac{\partial}{\partial p_{n \nu}}\right) I\left(p_{1}, \ldots, p_{n}\right)=0 .
$$

This equation can be contracted with all possible antisymmetric combinations of $p_{i \mu} p_{j \nu}$ to yield LI identities for $I$. For a three-point vertex, two of the external momenta are linearly independent $(n=$ 2 ), and only one antisymmetric combination of them can be constructed, resulting in one LI identity $\left(N_{\mathrm{LI}}=1\right)$. A four-point function depends on three external momenta $(n=3)$, allowing us to construct three linearly independent antisymmetric combinations, which yield three LI identities $\left(N_{\mathrm{LI}}=3\right)$. The full potential of the LI identities can only be exploited for integrals involving five or more external legs, which allow to construct six linearly independent antisymmetric combinations of external momenta, thus projecting out all six LI identities $\left(N_{\mathrm{LI}}=6\right)$.

Since $I$ is a scalar, it can not depend on the momenta $p_{i}$ itself, but only on scalar products $s_{i j}=2 p_{i} \cdot p_{j}$ of the external momenta. Replacing

$$
\frac{\partial}{\partial p_{i \mu}}=\sum_{j} 2\left(p_{i \mu}+p_{j \mu}\right) \frac{\partial}{\partial s_{i j}}
$$

one finds that (2.9) becomes a trivial identity, independent of $I$. However, the derivatives in (2.9) can be interchanged with the loop integrations in $I$, such that they do not act anymore on the integral $I$, but on the integrand of $I$. After this interchange, (2.9) becomes a non-trivial relation between different integrals.

In the case of two-loop four-point functions, one has a total of 13 equations (10 IBP $+3 \mathrm{LI})$ for each integrand corresponding to an integral of class $I_{t, r, s}$, relating integrals of the same topology with up to $s+1$ scalar products and $r+1$ denominators, plus integrals of simpler topologies (i.e. with a smaller number of different denominators). The 13 identities obtained starting from an integral $I_{t, r, s}$ do contain integrals of the following types:

- $I_{t, r, s}$ : the integral itself.

- $I_{t-1, r, s}:$ simpler topology.

- $I_{t, r+1, s}, I_{t, r+1, s+1}$ : same topology, more complicated than $I_{t, r, s}$.

- $I_{t, r-1, s}, I_{t, r-1, s-1}$ : same topology, simpler than $I_{t, r, s}$.

Quite in general, single identities of the above kind can be used to obtain the reduction of $I_{t, r+1, s+1}$ or $I_{t, r+1, s}$ integrals in terms of $I_{t, r, s}$ and simpler integrals - rather than to get information on the $I_{t, r, s}$ themselves.

Integrations-by-parts identities are widely applied in multi-loop calculations of inclusive quantities (see e.g. [4] for a review), which are related to two-point functions. In these calculations, only a relatively small number of different topologies has to be considered, but the integrals appearing in the calculation can bear large powers of propagators and scalar products, arising for example from expansions in masses or momenta. In these calculations, it is desirable to have reduction formulae for arbitrary powers of propagators and scalar products. These can be obtained from IBP identities derived for an integral with arbitrary powers (to be treated symbolically) of scalar products and propagators; the derivation of these symbolic reduction formulae requires a lot of ingenuity, based on the direct inspection of the explicit form of the IBP identities for each considered topology, and could not be carried out mechanically.

For loop integrals with a large number of external legs, IBP identities are needed for a large number of different topologies, but in general for relatively small powers of propagators and scalar products. In this 
case, it would therefore be desirable to have a mechanical procedure for solving, for any given topology, IBP and LI identities for integrals with fixed powers of the propagators and scalar products.

If one considers the set of all the identities obtained starting from the integrand of all the $N\left(I_{t, r, s}\right)$ integrals of class $I_{t, r, s}$, one obtains $\left(N_{\mathrm{IBP}}+N_{\mathrm{LI}}\right) N\left(I_{t, r, s}\right)$ identities which contain $N\left(I_{t, r+1, s+1}\right)+N\left(I_{t, r+1, s}\right)$ integrals of more complicated structure. From (2.2) it can be read off that with increasing $r$ and $s$ the number of identities grows faster than the number of new unknown integrals]. As a consequence, if for a given $t$-topology one considers the set of all the possible equations obtained by considering all the integrands up to certain values $r^{*}, s^{*}$ of $r, s$, for large enough $r^{*}, s^{*}$ the resulting system of equations is overconstrained and can be used for expressing the more complicated integrals, with greater values of $r, s$ in terms of simpler ones, with smaller values of $r, s$. (Let us observe that, the system being overconstrained, the equations cannot be all independent; it is not a priori known how many equations are in fact linearly independent and, correspondingly, how many integrals of the topology under consideration will remain after reduction).

The required values $r^{*}$ and $s^{*}$ for $r$ and $s$ can be found by counting the number of accumulated equations (equations for all integrals with $r \leq r^{*}$ and $s \leq s^{*}$ ) and comparing them with the number of accumulated unknown integrals, with $\left(r \leq r^{*}+1, s \leq s^{*}+1\right)$, but excluding $\left(r=t, s=s^{*}+1\right)$. As an example, we list in Table 1 the number of equations and unknowns for two-loop four-point functions with seven denominators $t=7$. It can be seen that a complete reduction requires at least one of the combinations $\left(r^{*}, s^{*}\right):(7,2) ;(8,1) ;(9,0)$.

$t=7$

different $I_{t, r, s}$

\begin{tabular}{|r||r|r|r|r|r|}
\hline$r$ & 0 & 1 & 2 & 3 & 4 \\
\hline \hline 7 & 1 & 2 & 3 & 4 & 5 \\
\hline 8 & 7 & 14 & 21 & 28 & 35 \\
\hline 9 & 28 & 56 & 84 & 112 & 140 \\
\hline 10 & 84 & 168 & 252 & 336 & 420 \\
\hline
\end{tabular}

\begin{tabular}{|c|c|c|c|c|}
\hline$s$ & 0 & 1 & 2 & 3 \\
\hline \multirow{2}{*}{7} & 13 & 39 & 78 & 130 \\
\hline & 22 & 45 & 76 & 115 \\
\hline \multirow{2}{*}{8} & 104 & 312 & 624 & 1040 \\
\hline & 106 & 213 & 354 & 535 \\
\hline \multirow{2}{*}{9} & 468 & 1404 & 2808 & 4680 \\
\hline & 358 & 717 & 1196 & 1795 \\
\hline
\end{tabular}

Table 1: Comparison of the number of (IBP and LI) identities to the number of new unknowns (different integrals with $t=7$ ) appearing in these equations for a two-loop box integral with $t=7$ internal propagators. The identities for $I_{t, r, s}$ contain at most $I_{t, r+1, s+1}$. It can be seen that for growing $r$ and $s$, the number of equations, upper number in each box, exceeds the number of unknowns, given by the lower number.

The above table illustrates that typically hundreds of equations have to be solved in order to obtain a reduction towards simpler integrals. The task is performed automatically (and independently of the topology!) by a computer program invoking repeatedly the computer algebra packages FORM [6] and MAPLE [7]. For any given four-point two-loop topology, this procedure can result either in a reduction towards a small number (typically one or two) of integrals of the topology under consideration and integrals of simpler topology (less different denominators), or even in a complete reduction of all integrals of the topology under consideration towards integrals with simpler topology. Left-over integrals of the topology under consideration are called irreducible master integrals or just master integrals. If a topology turns out to contain irreducible master integrals, one is in principle free to choose which integrals are taken as master integrals, as long as the chosen integrals are not related by the IBP and LI identities. In our reduction, we choose $I_{t, t, 0}$ for topologies with one master integral and $I_{t, t, 0}$ together with the required number of integrals of type $I_{t, t+1,0}$ for topologies with more than one master integral.

As a final point, it is worth noting that the procedure described above can be used to reduce a

\footnotetext{
${ }^{1}$ The importance of this fact was first pointed out by S. Laporta and exploited in [5].
} 
tensor integral towards scalar integrals. All integrals appearing in the projection of an arbitrary tensorial integral onto a tensor basis will take the form (2.1), i.e. they can be classified as $I_{t, r, s}$ and reduced to master integrals.

In the following section, we will demonstrate that the established reduction of all the integrals to a few master integrals can be used also to write differential equations in the external invariants $s_{i j}$ for the master integrals themselves, and then how the differential equations can be used to compute these master integrals.

\section{Differential Equations for Master Integrals}

The IBP and LI identities discussed in the previous section allow us to express integrals of the form (2.1) as a linear combination of a few master integrals, i.e. integrals which are not further reducible, but have to be computed by some different method.

For the case of massless two-loop four-point functions, several techniques have been proposed in the literature, such as for example the application of a Mellin-Barnes transformation to all propagators [8, 9] or the negative dimension approach [10]. Both techniques rely on an explicit integration over the loop momenta, with differences mainly in the representation used for the propagators. So far, these techniques were only applied to a limited number of master integrals: Smirnov [8] has recently used the Mellin-Barnes method to compute the planar double box integral for the case of all external legs on shell (massless case); the same method has been applied by Tausk [9] for the computation of the non-planar on-shell double box integral; the negative dimension approach has been applied by Anastasiou, Glover and Oleari 10 to compute the class of two-loop box integrals which correspond to a one-loop bubble insertion in one of the propagators of the one-loop box. A general method for the computation of the master integrals appearing in two-loop four-point functions has however not yet been found. So far, it has also not even been clear (apart from the planar double box topology, where Smirnov and Veretin have recently demonstrated the reducibility of any integral of this topology to two master integrals [11]) how many master integrals exist for a given topology. Solving the identities discussed in the previous section, we are now able to identify the irreducible master integrals. A list of reducible two-loop four-point topologies will be given in Section 5 .

A method for the computation of master integrals avoiding the explicit integration over the loop momenta is to derive differential equations in internal propagator masses or in external momenta for the master integral, and to solve these with appropriate boundary conditions. This method has first been suggested by Kotikov 12 to relate loop integrals with internal masses to massless loop integrals. It has been elaborated in detail and generalised to differential equations in external momenta in [13]; first applications were presented in [14]. In the case of four-point functions with one external off-shell leg and no internal masses, one has three independent invariants, resulting in three differential equations.

The derivatives in the invariants $s_{i j}=\left(p_{i}+p_{j}\right)^{2}$ can be expressed by derivatives in the external momenta:

$$
\begin{aligned}
& s_{12} \frac{\partial}{\partial s_{12}}=\frac{1}{2}\left(+p_{1}^{\mu} \frac{\partial}{\partial p_{1}^{\mu}}+p_{2}^{\mu} \frac{\partial}{\partial p_{2}^{\mu}}-p_{3}^{\mu} \frac{\partial}{\partial p_{3}^{\mu}}\right) \\
& s_{13} \frac{\partial}{\partial s_{13}}=\frac{1}{2}\left(+p_{1}^{\mu} \frac{\partial}{\partial p_{1}^{\mu}}-p_{2}^{\mu} \frac{\partial}{\partial p_{2}^{\mu}}+p_{3}^{\mu} \frac{\partial}{\partial p_{3}^{\mu}}\right) \\
& s_{23} \frac{\partial}{\partial s_{23}}=\frac{1}{2}\left(-p_{1}^{\mu} \frac{\partial}{\partial p_{1}^{\mu}}+p_{2}^{\mu} \frac{\partial}{\partial p_{2}^{\mu}}+p_{3}^{\mu} \frac{\partial}{\partial p_{3}^{\mu}}\right)
\end{aligned}
$$

The combinations of derivatives and momenta appearing on the right hand side of (3.1) are obviously linearly independent from the combinations appearing in the LI identities (2.9) (which vanish identically when acting on a function depending on the scalars $\left.s_{12}, s_{13}, s_{23}\right)$. The three derivatives of an integral $I_{t, r, s}\left(s_{12}, s_{13}, s_{23}, d\right)$ are not linearly independent, but related due to the properties of $I$ under rescaling of all external momenta:

$$
I_{t, r, s}\left(s_{12}, s_{13}, s_{23}, d\right)=\lambda^{-\alpha(d, r, s)} I_{t, r, s}\left(\lambda^{2} s_{12}, \lambda^{2} s_{13}, \lambda^{2} s_{23}, d\right),
$$


where $\alpha(d, r, s)$ is the mass dimension of the integral. For a $m$-loop integral in $d$ space-time dimensions with $r$ powers of denominators and $s$ scalar products, one finds $\alpha(d, r, s)=m d+2 s-2 r$. The above equation yields the rescaling relation

$$
\left[-\frac{\alpha}{2}+s_{12} \frac{\partial}{\partial s_{12}}+s_{13} \frac{\partial}{\partial s_{13}}+s_{23} \frac{\partial}{\partial s_{23}}\right] I_{t, r, s}\left(s_{12}, s_{13}, s_{23}, d\right)=0 .
$$

Let us now first consider the case of a topology with only one master integral, which is chosen to be $I_{t, t, 0}$, defined as

$$
I_{t, t, 0}\left(s_{i j}, s_{j k}, s_{k i}, d\right)=\int \frac{\mathrm{d}^{d} k}{(2 \pi)^{d}} \frac{\mathrm{d}^{d} l}{(2 \pi)^{d}} f_{t, t, 0}\left(k, l, p_{i}\right),
$$

where $f_{t, t, 0}\left(k, l, p_{i}\right)$ is a suitable integrand of the form appearing in (2.1). It is evident that acting with the right hand sides of (3.1) on the right hand side of (3.4) will, after interchange of derivative and integration, yield a a combination of integrals of the same type as appearing in the IBP and LI identities for $I_{t, t, 0}$, including integrals of type $I_{t, t+1,1}$ and $I_{t, t+1,0}$. Consequently, the scalar derivatives (on left hand side of (3.1)) of $I_{t, t, 0}$ can be expressed by a linear combination of integrals up to $I_{t, t+1,1}$ and $I_{t, t+1,0}$. These can all be reduced to $I_{t, t, 0}$ and to integrals of simpler topology by applying the IBP and LI identities. This reduction results in differential equations for $I_{t, t, 0}$ of the form:

$$
\begin{aligned}
s_{i j} \frac{\partial}{\partial s_{i j}} I_{t, t, 0}\left(s_{i j}, s_{j k}, s_{k i}, d\right)= & A\left(s_{i j}, s_{j k}, s_{k i}, d\right) I_{t, t, 0}\left(s_{i j}, s_{j k}, s_{k i}, d\right) \\
& +F\left(s_{i j}, s_{j k}, s_{k i}, d, I_{t-1, r, s}\left(s_{i j}, s_{j k}, s_{k i}, d\right)\right),
\end{aligned}
$$

where $s_{i j}, s_{j k}, s_{k i}$ are the three invariants, $A\left(s_{i j}, s_{j k}, s_{k i}, d\right)$ turns out to be a rational function of the invariants and of $d, F\left(I_{t-1, r, s}\right)$ is a linear combination (with coefficients depending on $s_{i j}, s_{j k}, s_{k i}$ and $d$ ) of integrals of type $I_{t-1, r, s}$, containing only topologies simpler than $I_{t, t, 0}$, but potentially with high powers of some denominators and scalar products. These $I_{t-1, r, s}$, which refer to a simpler topology and therefore can be considered as known in a bottom-up approach, play the role of an inhomogeneous term in the equation; one can then look for the proper solution of (3.5) in a straightforward way.

The master integral $I_{t, t, 0}\left(s_{i j}, s_{j k}, s_{k i}, d\right)$ can indeed be obtained by matching the general solution of (3.5) to an appropriate boundary condition. Quite in general, finding a boundary condition is a simpler problem than evaluating the whole integral, since it depends on a smaller number of kinematical variables. In some cases, the boundary condition can even be determined from the differential equation itself: for $s_{i j}=0$, 3.5 yields, if $A\left(0, s_{j k}, s_{k i}, d\right) \neq 0$,

$$
I_{t, t, 0}\left(0, s_{j k}, s_{k i}, d\right)=-\left[A\left(0, s_{j k}, s_{k i}, d\right)\right]^{-1} F\left(0, s_{j k}, s_{k i}, d, I_{t-1, r, s}\left(0, s_{j k}, s_{k i}, d\right)\right) .
$$

For $A$ vanishing at $s_{i j}=0$ one can consider $I_{t, t, 0}$ in the limit where one of the external momenta vanishes, corresponding to the vanishing of both invariants involving this momentum, e.g. $s_{i j}=0$ and $s_{k i}=0$ for $p_{i}^{\mu}=0$. In this case, $I_{t, t, 0}$ reduces to a three-point vertex function with one off-shell external leg. All these functions, which might be determined by iterating the procedure just described for the considered 4-point function, have actually already been computed at the two-loop level in 15 using IBP identities to reduce all possible topologies to a few master integrals, which, in this case, can be computed straightforwardly using Feynman parameters (cf. Section 5). Starting from the boundary condition in $s_{i j}=s_{k i}=0$, one can determine $I_{t, t, 0}\left(0, s_{j k}, s_{k i}, d\right)$ by solving the differential equation in $s_{k i}-$ this provides the desired boundary condition in $s_{i j}=0$.

For topologies with more than one master integral, 3.5 will be replaced by a system of coupled, linear, first order differential equations for all master integrals of the topology under consideration. The determination of the master integrals from these equations follows the same lines as discussed above, with the only difference that the general solution for the system of coupled differential equations is harder to obtain than for a single equation. In case the coupled equations can not be decoupled by an appropriate choice of variables, several mathematical techniques can be employed here $[16]$ : the system of $n$ coupled first order differential equations can for example be rewritten into one $n$-th order differential equation, 
which is then solved with standard methods. In some cases, the system can also be transformed into a form which is known to be solved by generalised hypergeometric series [16, 17, 18, 19].

It is clear from the above discussion, that the determination of a master integral of a certain topology with $t$ different denominators requires the knowledge of all the integrals appearing in the inhomogeneous term. These integrals are subtopologies of the topology of the integral under consideration, and contain at most $t-1$ different denominators. The determination of master integrals has therefore to proceed bottomup from simpler topologies with a small number of different denominators towards more complicated topologies with an increasing number of different denominators. For the case under special consideration, massless two-loop four-point functions with up to one external leg off-shell, this implies that one has to progress from the simplest master integrals with $t=3$ (off-shell two-point function) to construct all master integrals up to $t=7$.

\section{A Pedagogical Example: the One-Loop Four-Point Function}

To illustrate how the method explained above works in practice, we present in this section a detailed and self-contained derivation of the one-loop four-point function

\begin{tabular}{c|l|l}
$\vec{q}$ & & $p_{2}$ \\
$p_{1}$ & & $p_{3}$
\end{tabular}$=\int \frac{\mathrm{d}^{d} k}{(2 \pi)^{d}} \frac{1}{k^{2}\left(k-p_{2}\right)^{2}\left(k-p_{2}-p_{3}\right)^{2}\left(k-p_{1}-p_{2}-p_{3}\right)^{2}}$.

The topology of this integral is given by the set of its four propagators, it is the only $t=4$ topology at one loop. In the notation introduced above, (4.1) corresponds to an integral $I_{4,4,0}$, the simplest integral of this topology with all propagators appearing in first power and with no scalar products. For the one-loop four-point function, one has four independent scalar products involving $k_{\mu}\left(k^{2}\right.$ and $\left.k_{\mu} p_{i}^{\mu}\right)$ and four linear independent denominators. This implies that any scalar product involving $k_{\mu}$ can be rewritten as linear combination of propagators and invariants $s_{i j} \equiv 2 p_{i \mu} p_{j}^{\mu}$. Consequently, no integrals with scalar products in the numerator can appear for this topology.

The reduction of all four integrals with one squared propagator $\left(I_{4,5,0}\right)$ can be carried out by considering the IBP identities for $I_{4,4,0}$ :

$$
\int \frac{\mathrm{d}^{d} k}{(2 \pi)^{d}} \frac{\partial}{\partial k^{\mu}} \frac{v^{\mu}}{k^{2}\left(k-p_{2}\right)^{2}\left(k-p_{2}-p_{3}\right)^{2}\left(k-p_{1}-p_{2}-p_{3}\right)^{2}}=0,
$$

where $v^{\mu}$ can be the loop momentum $k^{\mu}$ or any of the external momenta $p_{i}^{\mu}$, thus yielding four identities. It turns out that LI identities and IBP identities for integrals with higher powers of the propagators do not contain additional information which would allow a reduction of $I_{4,4,0}$. The integral (4.1) is therefore a master integral. It is the only master integral for this topology.

To proceed towards the differential equations in the invariants $s_{12}, s_{13}$ and $s_{23}$ for this master integral, let us consider the derivatives in the external momenta:

$$
\begin{aligned}
& p_{1}^{\mu} \frac{\partial}{\partial p_{1}^{\mu}} \quad \begin{array}{l}
\vec{q} \\
p_{1}
\end{array} \quad\left[\begin{array}{l}
p_{2} \\
p_{3}
\end{array}=\int \frac{\mathrm{d}^{d} k}{(2 \pi)^{d}} \frac{1}{k^{2}\left(k-p_{2}\right)^{2}\left(k-p_{2}-p_{3}\right)^{2}\left(k-p_{1}-p_{2}-p_{3}\right)^{2}}\right. \\
& \left(\frac{2 p_{1}^{\mu}\left(k-p_{1}-p_{2}-p_{3}\right)_{\mu}}{\left(k-p_{1}-p_{2}-p_{3}\right)^{2}}\right), \\
& p_{2}^{\mu} \frac{\partial}{\partial p_{2}^{\mu}} \quad \begin{array}{l}
\vec{q} \\
p_{1}
\end{array} \quad \quad \begin{array}{l}
p_{2} \\
p_{3}
\end{array}=\int \frac{\mathrm{d}^{d} k}{(2 \pi)^{d}} \frac{1}{k^{2}\left(k-p_{2}\right)^{2}\left(k-p_{2}-p_{3}\right)^{2}\left(k-p_{1}-p_{2}-p_{3}\right)^{2}} \\
& \left(\frac{2 p_{2}^{\mu}\left(k-p_{1}-p_{2}-p_{3}\right)_{\mu}}{\left(k-p_{1}-p_{2}-p_{3}\right)^{2}}+\frac{2 p_{2}^{\mu}\left(k-p_{2}-p_{3}\right)_{\mu}}{\left(k-p_{2}-p_{3}\right)^{2}}+\frac{2 p_{2}^{\mu}\left(k-p_{2}\right)_{\mu}}{\left(k-p_{2}\right)^{2}}\right), \\
& \begin{array}{cc|c|c}
p_{3}^{\mu} \frac{\partial}{\partial p_{3}^{\mu}} & \vec{q} \\
p_{1}
\end{array} \quad \mid \begin{array}{l}
p_{2} \\
p_{3}
\end{array}=\int \frac{\mathrm{d}^{d} k}{(2 \pi)^{d}} \frac{1}{k^{2}\left(k-p_{2}\right)^{2}\left(k-p_{2}-p_{3}\right)^{2}\left(k-p_{1}-p_{2}-p_{3}\right)^{2}}
\end{aligned}
$$




$$
\left(\frac{2 p_{3}^{\mu}\left(k-p_{1}-p_{2}-p_{3}\right)_{\mu}}{\left(k-p_{1}-p_{2}-p_{3}\right)^{2}}+\frac{2 p_{3}^{\mu}\left(k-p_{2}-p_{3}\right)_{\mu}}{\left(k-p_{2}-p_{3}\right)^{2}}\right) .
$$

The right hand sides of the above equations contain terms with

(i) four different propagators with one squared propagator, no scalar product

(ii) four different propagators, no squared propagator, no scalar product

(iii) three different propagators, one squared propagator.

The terms of type (i) can now be reduced to type (ii) and (iii) by using the integration-by-parts identities (4.2). One obtains:

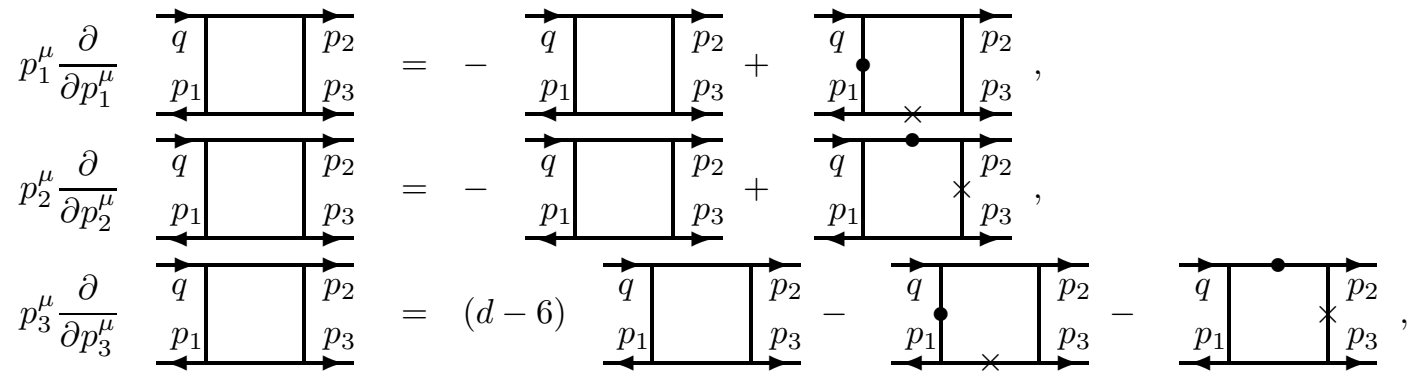

where $(\bullet)$ denotes a squared propagator and $(\times)$ stands for a pinched (cancelled) propagator.

A check on these equations is provided by the rescaling relation (3.3)

$$
\left.\left[4-\frac{d}{2}+s_{12} \frac{\partial}{\partial s_{12}}+s_{13} \frac{\partial}{\partial s_{13}}+s_{23} \frac{\partial}{\partial s_{23}}\right] \quad \begin{array}{l|l|l}
\vec{q} \\
p_{1}
\end{array}\right] \quad \begin{aligned}
& \overrightarrow{p_{2}} \\
& p_{3}
\end{aligned}=0,
$$

which is related to the above equations by

$$
s_{12} \frac{\partial}{\partial s_{12}}+s_{13} \frac{\partial}{\partial s_{13}}+s_{23} \frac{\partial}{\partial s_{23}}=\frac{1}{2} \sum_{i=1}^{3} p_{i}^{\mu} \frac{\partial}{\partial p_{i}^{\mu}} .
$$

Inserting the derivatives obtained above, one finds that the rescaling relation is indeed fulfilled.

The three-propagator terms appearing in the above equations can be further reduced by using the IBP identities for the corresponding vertex; the vertex amplitudes, in turn, can all be expressed in terms of bubble integrals, and one finally finds

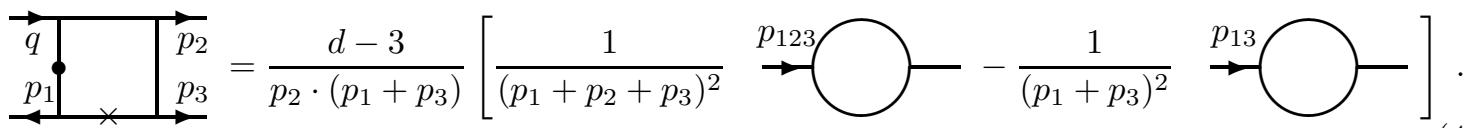

A similar identity is obtained by exchanging $p_{1} \leftrightarrow p_{2}$.

The differential equations for the one-loop box integral then follow from (3.1). The set of differential equations reads:

$$
\begin{aligned}
& s_{12} \frac{\partial}{\partial s_{12}} \quad \begin{array}{l}
\vec{q} \\
p_{1}
\end{array}|\quad| \begin{array}{ll}
p_{2} \\
p_{3}
\end{array}=-\frac{d-4}{2} \quad \begin{array}{l}
\vec{q} \\
\boldsymbol{p}_{1}
\end{array}=\mid \begin{array}{l}
p_{2} \\
p_{3}
\end{array} \\
& +\frac{2(d-3)}{s_{12}+s_{13}}\left[\frac{1}{s_{123}} \stackrel{p_{123}}{\rightarrow} \bigcirc-\frac{1}{s_{23}} \stackrel{p_{23}}{\longrightarrow} \square-\right] \\
& +\frac{2(d-3)}{s_{12}+s_{23}}\left[\frac{1}{s_{123}} \stackrel{p_{123}}{\rightarrow} \bigcirc-\frac{1}{s_{13}} \stackrel{p_{13}}{\longrightarrow} \square-\right.
\end{aligned}
$$




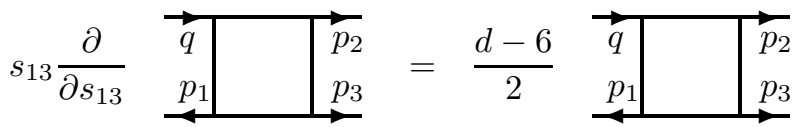

$$
\begin{aligned}
& -\frac{2(d-3)}{s_{12}+s_{13}}\left[\frac{1}{s_{123}} \stackrel{p_{123}}{\rightarrow}\right)-\frac{1}{s_{23}} \stackrel{p_{23}}{\longrightarrow} \square-
\end{aligned}
$$

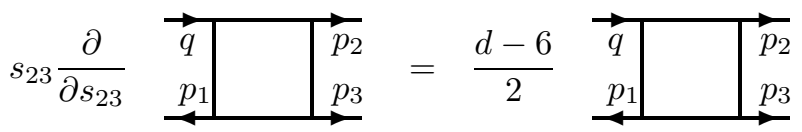

$$
\begin{aligned}
& -\frac{2(d-3)}{s_{12}+s_{23}}\left[\frac{1}{s_{123}} \stackrel{p_{123}}{\rightarrow} \longrightarrow-\frac{1}{s_{13}} \stackrel{p_{13}}{\longrightarrow} \square\right.
\end{aligned}
$$

where $s_{123}=s_{12}+s_{13}+s_{23}$. The one-loop bubble diagrams in the inhomogenous term yield:

$$
\stackrel{p}{\rightarrow}=\left[\frac{(4 \pi)^{\frac{4-d}{2}}}{16 \pi^{2}} \frac{\Gamma(3-d / 2) \Gamma^{2}(d / 2-1)}{\Gamma(d-3)}\right] \frac{-2 i}{(d-4)(d-3)}\left(-p^{2}\right)^{\frac{d-4}{2}} \equiv A_{2, L O}\left(-p^{2}\right)^{\frac{d-4}{2}} .
$$

The boundary conditions in $s_{i j}=0$ can be readily read off from the above:

$$
\begin{array}{l|l|l}
\vec{q} \\
p_{1}
\end{array} \quad \begin{aligned}
& p_{3} \\
& p_{3}
\end{aligned}\left(s_{12}=0\right)=\frac{4(d-3)}{(d-4)} \frac{1}{s_{13} s_{23}} \stackrel{p_{123}}{\longrightarrow} \longrightarrow
$$
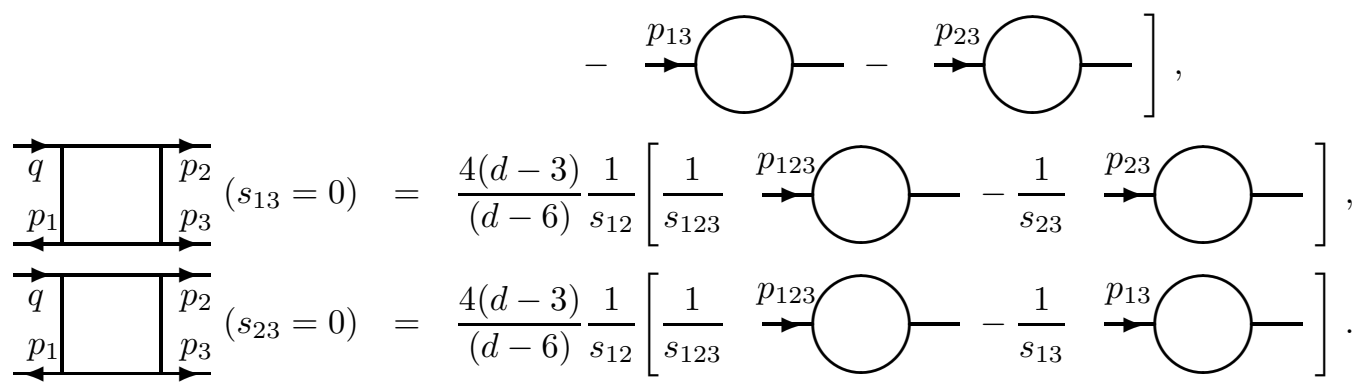

The result for the one-loop box integral can in principle be obtained by integrating any of the differential equations (4.9)-(4.11). In practice, it turns out to be more appropriate to introduce a new set of variables, namely $s_{13}, s_{23}$ and $s_{123}=s_{12}+s_{13}+s_{23}$, corresponding to the arguments appearing in the two-point functions in the inhomogeneous terms. This transformation yields a differential equation in $s_{123}$, which will be used for integration. Note that this transformation also modifies the differential equations in $s_{13}$ and $s_{23}$. The differential equation in $s_{123}$ reads:

$$
\begin{aligned}
& \left.\left.\begin{array}{cc|c|cc|c|c}
\partial & \vec{q} \\
\partial s_{123} & p_{1}
\end{array}\right] \quad \begin{array}{ll}
p_{2} \\
p_{3}
\end{array}+\frac{d-4}{2\left(s_{123}-s_{13}-s_{23}\right)} \quad \begin{array}{l}
\vec{q} \\
\vec{p}_{1}
\end{array}\right] \quad \begin{array}{l}
p_{2} \\
p_{3}
\end{array}= \\
& +\frac{2(d-3)}{\left(s_{123}-s_{23}\right)\left(s_{123}-s_{13}-s_{23}\right)}\left[\frac{1}{s_{123}} \stackrel{p_{123}}{\rightarrow}--\frac{1}{s_{23}} \stackrel{p_{23}}{\longrightarrow} \square\right. \\
& \left.+\frac{2(d-3)}{\left(s_{123}-s_{13}\right)\left(s_{123}-s_{13}-s_{23}\right)}\left[\frac{1}{s_{123}} \stackrel{p_{123}}{\rightarrow}\right)-\frac{1}{s_{13}} \stackrel{p_{13}}{\longrightarrow} \square-\right] .
\end{aligned}
$$

The boundary condition in $s_{123}=0$ can not be trivially determined from this equation, reflecting the fact that the massless one-loop box integral with all external legs on shell is not reducible to simpler subtopologies by IBP identities. The boundary condition in $s_{123}=-s_{13}-s_{23}$ can however be determined from (4.13).

Equation (4.16) is a linear, inhomogeneous first order differential equation of the form

$$
\frac{\partial y(x)}{\partial x}+f(x) y(x)=g(x)
$$


which can be solved by introducing an integrating factor (see for instance [20] or any standard book on differential equations)

$$
M(x)=e^{\int f(x) \mathrm{d} x},
$$

such that $y(x)=1 / M(x)$ solves the homogenous differential equation $(g(x)=0)$. This yields the general solution of the inhomogenous equation as

$$
y(x)=\frac{1}{M(x)}\left(\int g(x) M(x) \mathrm{d} x+C\right),
$$

where the integration constant $C$ can be adjusted to match the boundary conditions.

For $(4.16)$, we have at once the integrating factor

$$
M\left(s_{123}\right)=\left(s_{13}+s_{23}-s_{123}\right)^{\frac{d-4}{2}} .
$$

This factor is not unambiguous, since

$$
M^{\prime}\left(s_{123}\right)=\left(s_{123}-s_{13}-s_{23}\right)^{\frac{d-4}{2}} .
$$

would also be a valid integrating factor. We select 4.17) by requiring a real integrating factor in the region: $-s_{123} \geq-s_{13}-s_{23} \geq 0$. The final result for the box integral does not depend on the selection of the integrating factor; using (4.18), one must however be more careful in applying analytic continuation formulae and in multiplying non-integer powers of the invariants.

With the integrating factor (4.17), the one-loop box integral reads:

$$
\begin{aligned}
& \begin{array}{l|l|l}
\vec{q} \\
p_{1}
\end{array} \quad \begin{array}{l}
p_{2} \\
\underbrace{}_{3}
\end{array}\left(s_{123}, s_{13}, s_{23}\right)=2(d-3) A_{2, L O}\left(s_{13}+s_{23}-s_{123}\right)^{2-\frac{d}{2}} \int^{s_{123}} \mathrm{~d} s_{123}^{\prime}\left(s_{13}+s_{23}-s_{123}^{\prime}\right)^{\frac{d}{2}-3} \\
& {\left[\frac{\left(-s_{13}\right)^{\frac{d}{2}-3}}{s_{13}-s_{123}^{\prime}}+\frac{\left(-s_{23}\right)^{\frac{d}{2}-3}}{s_{23}-s_{123}^{\prime}}-\frac{2 s_{123}^{\prime}-s_{13}-s_{23}}{\left(s_{13}-s_{123}^{\prime}\right)\left(s_{23}-s_{123}^{\prime}\right)}\left(-s_{123}^{\prime}\right)^{\frac{d}{2}-3}\right] .}
\end{aligned}
$$

From a computational point of view, the evaluation of the box amplitude of (4.1) has been reduced to the one dimensional integration corresponding to the solution of (4.16).

The lower boundary of the integral is independent of $s_{123}$ and can be adjusted arbitrarily. The first two terms in (4.19) can be easily integrated by shifting the integration variable to $s_{123}^{\prime}-s_{13}-s_{23}$, which is then integrated between 0 and $s_{123}-s_{13}-s_{23}$. To integrate the last term, one introduces a new variable $s_{123}^{\prime}\left(s_{123}^{\prime}-s_{13}-s_{23}\right)$, which is integrated between 0 and $s_{123}\left(s_{123}-s_{13}-s_{23}\right)$. The resulting integrals yield can be identified as integral representation of the hypergeometric function ${ }_{2} F_{1}$. With this choice of variables and boundaries, no constant term is required to match the boundary conditions. The result for the one-loop box integral then reads:

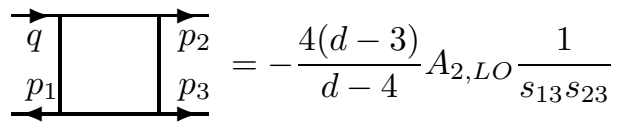

$$
\begin{aligned}
& {\left[\left(\frac{s_{13} s_{23}}{s_{13}-s_{123}}\right)^{\frac{d}{2}-2}{ }_{2} F_{1}\left(d / 2-2, d / 2-2 ; d / 2-1 ; \frac{s_{123}-s_{13}-s_{23}}{s_{123}-s_{13}}\right)\right.} \\
& +\left(\frac{s_{13} s_{23}}{s_{23}-s_{123}}\right)^{\frac{d}{2}-2}{ }_{2} F_{1}\left(d / 2-2, d / 2-2 ; d / 2-1 ; \frac{s_{123}-s_{13}-s_{23}}{s_{123}-s_{23}}\right) \\
& \left.-\left(\frac{-s_{123} s_{13} s_{23}}{\left(s_{13}-s_{123}\right)\left(s_{23}-s_{123}\right)}\right)^{\frac{d}{2}-2}{ }_{2} F_{1}\left(d / 2-2, d / 2-2 ; d / 2-1 ; \frac{s_{123}\left(s_{123}-s_{13}-s_{23}\right)}{\left(s_{123}-s_{13}\right)\left(s_{123}-s_{23}\right)}\right)\right] \text {. }
\end{aligned}
$$


The invariants appearing in this expression can be safely continued from the region $-s_{123} \geq-s_{13}-s_{23} \geq 0$ to the physical region $s_{123} \geq s_{13}+s_{23} \geq 0$. The arguments of the hypergeometric functions are ratios of invariants, they are not changed by the analytic continuation. The non-integer powers of invariants appearing as coefficients acquire imaginary parts, their signs are uniquely determined by the convention $-p^{2}=-p^{2}-i 0$, thus fixing the imaginary part of the whole expression. The above equation reproduces the well-known result from the literature, e.g. [21]. It should be kept in mind that in applying dimensional regularisation, no distinction between infrared and ultraviolet poles, which both show up as $1 /(d-4)$ in the above equation and in the coefficient $A_{2, L O}$, is made. Needless to say, those singularities are an intrinsic feature of the dimensional regularisation, and by non means an artifact of the differential equation approach. In particular, the most singular part of the above one loop integral, which is ultraviolet finite, as well as of the two loop integrals discussed in the following section, arises from soft configurations, and can in principle be re-derived by applying a strong ordering procedure [22 to the integrands.

When the box integral is expressed in the above form, where no expansion around $d=4$ has yet been performed, analytic continuations, e.g. to the on-shell case $s_{123}=0$ or to collinear and soft limits $s_{i j}=0$, can be made with ease.

\section{Results on Two-Loop Four-Point Functions}

In the following, we shall outline how the techniques derived in Sections 2 and 3 can be applied to the computation of two-loop integrals appearing in amplitudes for the decay of one massive into three massless particles: two-loop four-point functions with one off-shell leg. The main purpose of this section is to illustrate applications of the tools developed above to non-trivial problems; the list of integrals given here is far from complete. We provide a comprehensive list of master integrals only up to $t=5$, for $t=6$ and $t=7$ only reducible integrals are quoted.

A prefactor common to all massless scalar integrals is

$$
S_{d}=\left[(4 \pi)^{\frac{4-d}{2}} \frac{\Gamma(3-d / 2) \Gamma^{2}(d / 2-1)}{\Gamma(d-3)}\right],
$$

which is also appearing in all counterterms in the $\overline{\mathrm{MS}}-$-scheme.

In the following, the notation of external momenta is as follows: $p_{i}$ denotes an on-shell momentum, $p_{i j(k)}$ denotes an off-shell momentum, being the sum of two (three) on-shell momenta $p_{i}, p_{j}\left(, p_{k}\right)$ with $s_{i j(k)}=\left(p_{i j(k)}\right)^{2} \cdot p$ is an arbitrary momentum.

\section{$5.1 \quad t=3$}

For two-loop integrals with $t=3$, only one topology exists: the two-loop vacuum bubble. The corresponding integral fulfils a homogeneous differential equation, which can not be used to infer any boundary condition. The integral can however be computed using Feynman parameters:

$$
\begin{aligned}
\stackrel{p}{\rightarrow} & =\left(\frac{S_{d}}{16 \pi^{2}}\right)^{2} \frac{\Gamma(5-d) \Gamma^{2}(d-3)}{\Gamma^{2}(3-d / 2) \Gamma(d / 2-1) \Gamma(3 d / 2-3)} \frac{1}{(d-3)(d-4)}\left(-p^{2}\right)^{d-3} \\
& \equiv A_{3}\left(-p^{2}\right)^{d-3} .
\end{aligned}
$$

\section{$5.2 t=4$}

Several different two-loop topologies exist for $t=4$. Two types of two-point functions are encountered. The first can be reduced to

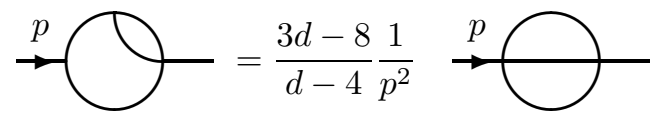


using IBP identities. The second is the product of two one-loop bubble integrals and yields

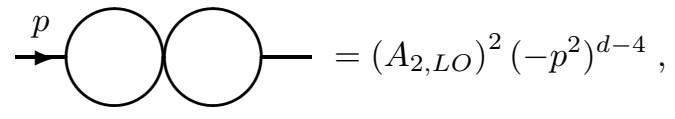

which generalises trivially to a three-point function

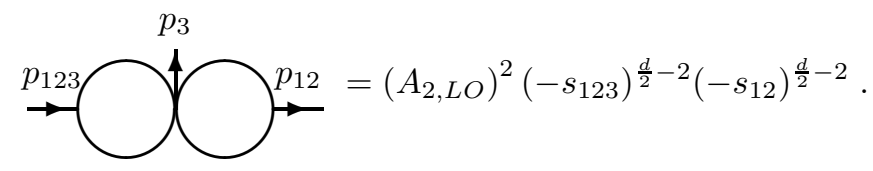

Only one of the master integrals at $t=4$ fulfils a homogeneous differential equation: a two-loop vertex integral with one off-shell leg. This integral can also be computed using Feynman parameters:

$$
\begin{aligned}
\stackrel{p_{12}}{\longrightarrow} \coprod_{p_{2}}^{p_{1}} & =\left(\frac{S_{d}}{16 \pi^{2}}\right)^{2} \frac{\Gamma^{3}(d-3) \Gamma(5-d)}{\Gamma(3-d / 2) \Gamma^{2}(d / 2-1) \Gamma(3 d / 2-4)} \frac{-2}{(d-3)(d-4)^{2}}\left(-s_{12}\right)^{d-4} \\
& \equiv A_{4}\left(-s_{12}\right)^{d-4} .
\end{aligned}
$$

The other vertex integral topology with one off-shell leg can be reduced using IBP identities:

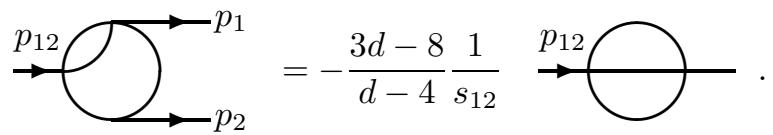

Among the three vertex integrals with two off-shell legs, only one can be reduced using IBP and LI identities:

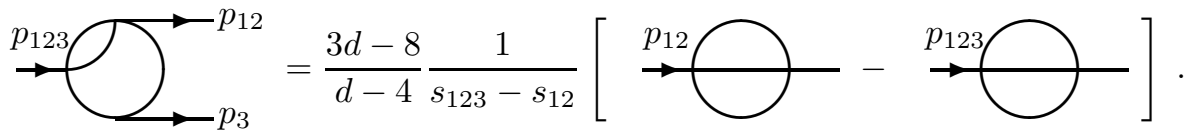

The two remaining ones are master integrals. Written out in terms of propagators, they read:

$$
\begin{aligned}
& \longrightarrow{ }_{\longrightarrow}^{p_{123}}=\int \frac{\mathrm{d}^{d} k}{(2 \pi)^{d}} \frac{\mathrm{d}^{d} l}{(2 \pi)^{d}} \frac{1}{k^{2} l^{2}\left(k-p_{123}\right)^{2}\left(k-l-p_{12}\right)^{2}}, \\
& \longrightarrow \mathrm{P}_{12}^{\longrightarrow}=\int \frac{\mathrm{d}^{d} k}{(2 \pi)^{d}} \frac{\mathrm{d}^{d} l}{(2 \pi)^{d}} \frac{1}{k^{2} l^{2}\left(l-p_{12}\right)^{2}\left(k-l-p_{3}\right)^{2}} .
\end{aligned}
$$

Both fulfil inhomogeneous differential equations. For a vertex $p_{123} \rightarrow p_{12}+p_{3}$, the appropriate variables for the differential equations are $s_{123}$ and $s_{12}$. To illustrate the structure of the differential equations, we quote them for (5.9):

$$
\begin{aligned}
& s_{123} \frac{\partial}{\partial s_{123}} \stackrel{p_{123}}{\rightarrow} \longrightarrow^{p_{12}}=\frac{d-4}{2} \frac{2 s_{123}-s_{12}}{s_{123}-s_{12}} \stackrel{p_{123}}{\rightarrow} \longrightarrow_{-p_{3}}^{p_{12}} \\
& -\frac{3 d-8}{2} \frac{1}{s_{123}-s_{12}} \stackrel{p_{12}}{\rightarrow} \longrightarrow \\
& s_{12} \frac{\partial}{\partial s_{12}} \stackrel{p_{123}}{\rightarrow} \longrightarrow_{\longrightarrow p_{3}}^{p_{12}}=-\frac{d-4}{2} \frac{s_{12}}{s_{123}-s_{12}} \stackrel{p_{123}}{\longrightarrow}{ }^{-p_{12}}
\end{aligned}
$$




$$
+\frac{3 d-8}{2} \frac{1}{s_{123}-s_{12}} \stackrel{p_{12}}{\longrightarrow} \longrightarrow
$$

The boundary conditions for $s_{123}=0$ or $s_{12}=0$ are obtained directly from the vertex integrals with one off-shell leg quoted above. Using these, one finds

$$
\stackrel{p_{123}}{\longrightarrow} \bigcap_{-p_{3}}^{p_{12}}=A_{4}\left(s_{12}-s_{123}\right)^{\frac{d}{2}-2}\left(-s_{123}\right)^{\frac{d}{2}-2}-\frac{3 d-8}{2(d-3)} A_{3} \frac{\left(-s_{12}\right)^{d-3}}{-s_{123}}{ }_{2} F_{1}\left(\frac{d}{2}-1,1 ; d-2 ; \frac{s_{12}}{s_{123}}\right) \text {. }
$$

The second master integral can be obtained from this by analytic continuation of the hypergeometric function:

$$
\longrightarrow_{\longrightarrow p_{12}}^{p_{123}}=-\frac{3 d-8}{d-4} A_{3}\left(-s_{12}\right)^{\frac{d}{2}-2}\left(-s_{123}\right)^{\frac{d}{2}-2}{ }_{2} F_{1}\left(\frac{d}{2}-1,2-\frac{d}{2} ; 3-\frac{d}{2} ; \frac{s_{123}-s_{12}}{s_{123}}\right) .
$$

Vertex integrals with three off-shell legs can not appear as subtopologies in two-loop four-point functions with one off-shell leg.

\section{$5.3 t=5$}

The two-loop two-point function with $t=5$ is a well known example [3, 何 for the application of IBP identities:

$$
\stackrel{p}{\rightarrow}=\frac{2(3 d-8)(3 d-10)}{(d-4)^{2}} \frac{1}{\left(p^{2}\right)^{2}} \stackrel{p}{\rightarrow} \longrightarrow-\frac{2(d-3)}{d-4} \frac{1}{p^{2}} \stackrel{p}{\rightarrow}
$$

The four different $t=5$ three-point functions with one off-shell leg can also be reduced by using IBP and LI identities:
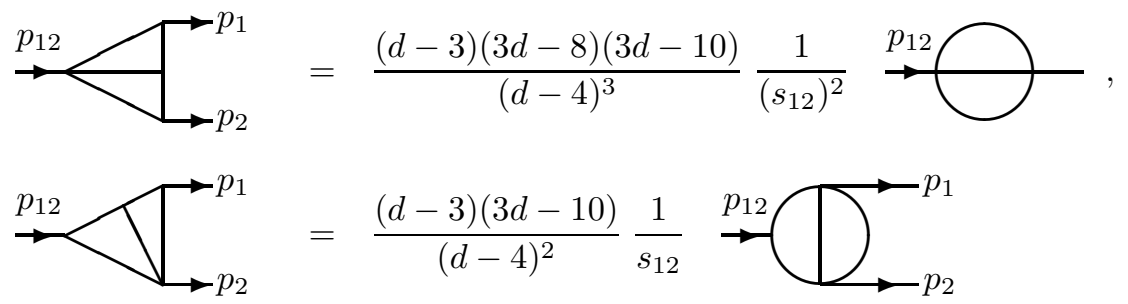

$$
-\frac{(d-3)(3 d-8)(3 d-10)}{(d-4)^{3}} \frac{1}{\left(s_{12}\right)^{2}} \stackrel{p_{12}}{\longrightarrow} \longrightarrow
$$

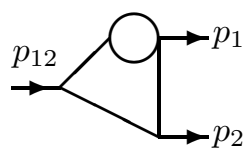

$$
=-\frac{(3 d-8)(3 d-10)}{(d-4)^{2}} \frac{1}{\left(s_{12}\right)^{2}} \stackrel{p_{12}}{\longrightarrow}
$$

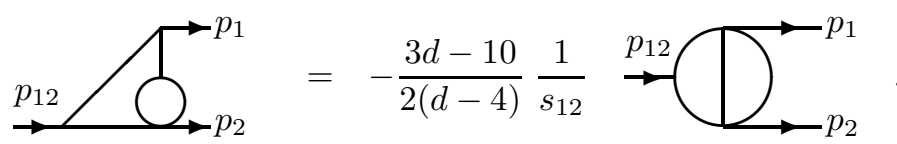

By applying IBP and LI identities, it is likewise possible to reduce all but one $t=5$ three-point function with two off-shell legs:

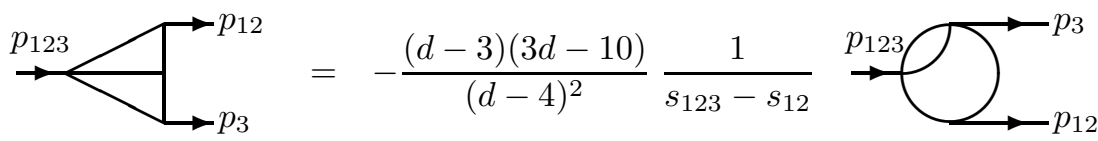




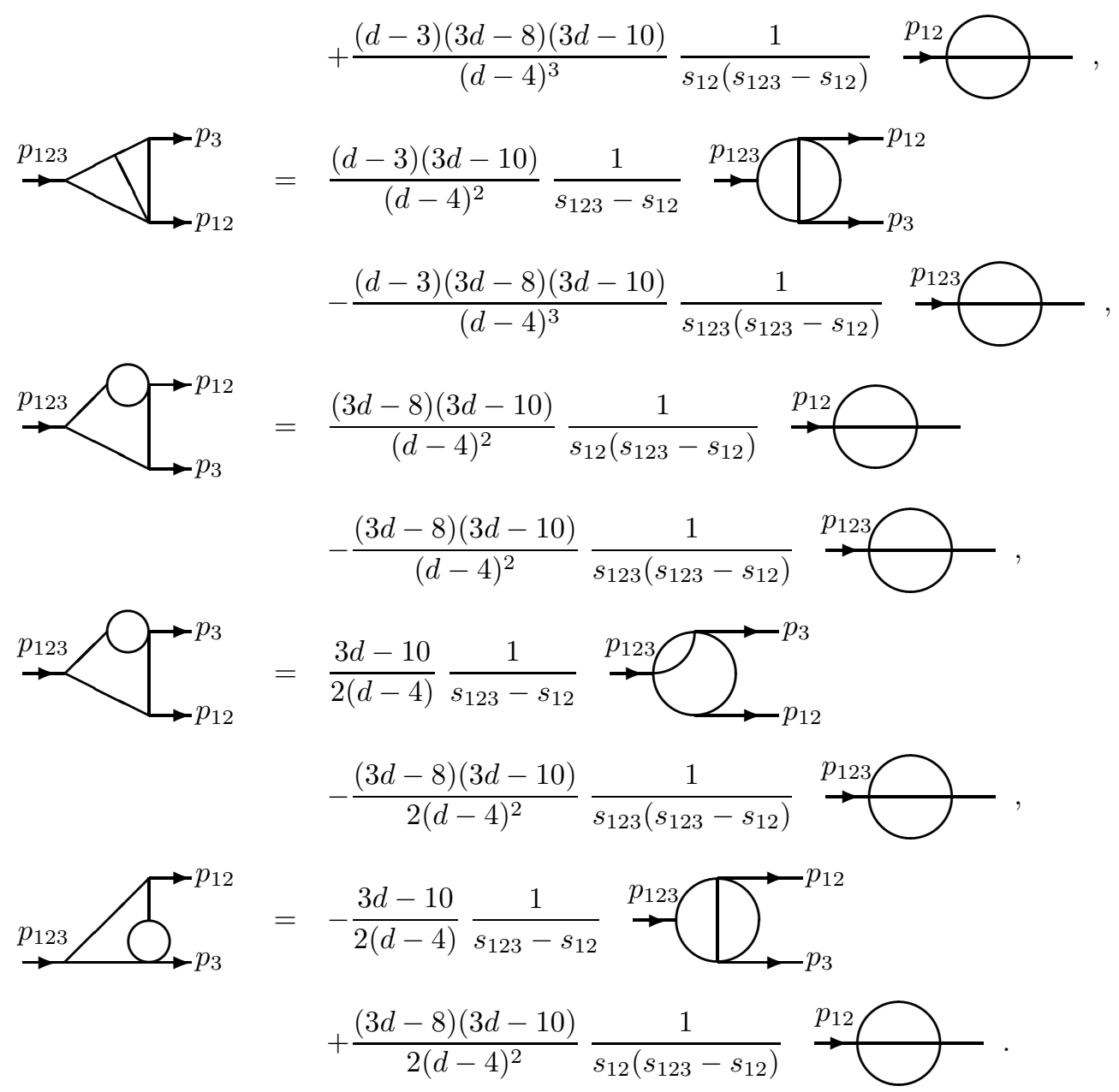

The remaining three-point function is a master integral, which can be found by solving the corresponding differential equations:

$$
\begin{aligned}
\longrightarrow p_{3}= & \frac{2(d-3)}{d-4}\left(A_{2, L O}\right)^{2}\left(-s_{12}\right)^{d-4} \frac{1}{-s_{123}}{ }_{2} F_{1}\left(1-\frac{d}{2}, d-3 ; d-2 ; \frac{s_{123}-s_{12}}{s_{123}}\right) \\
& -\frac{(3 d-8)(3 d-10)}{(d-4)^{2}} A_{3} \\
& {\left[\frac{1}{s_{12}}\left(-s_{123}\right)^{d-4}{ }_{3} F_{2}\left(\frac{d}{2}-1,1, d-3 ; 3-\frac{d}{2}, d-2 ; \frac{s_{12}-s_{123}}{s_{12}}\right)\right.} \\
& \left.+\frac{1}{s_{123}}\left(-s_{12}\right)^{d-4}{ }_{3} F_{2}\left(\frac{d}{2}-1,1, d-3 ; 3-\frac{d}{2}, d-2 ; \frac{s_{123}-s_{12}}{s_{123}}\right)\right] .
\end{aligned}
$$

For $t=5$, one finds four different topologies for two-loop four-point functions. These are all master integrals obeying inhomogeneous differential equations in the external invariants. Solving these equations and matching the boundary conditions, all master integrals can be determined.

$$
{ }_{p_{1} \rightarrow \mathrm{P}_{123} \rightarrow p_{3}} \mathrm{p}_{2}=\frac{3 d-10}{d-4} A_{4} \frac{1}{s_{23}}\left(-s_{13}\right)^{d-4}\left(\frac{s_{12}+s_{13}}{s_{23}}\right)^{3-d}
$$




$$
\begin{aligned}
& { }_{2} F_{1}\left(d-3, \frac{d}{2}-2 ; \frac{d}{2}-1 ; \frac{s_{12}}{s_{12}+s_{13}}\right) \\
& -\frac{3 d-10}{2(d-4)} A_{4} \frac{1}{s_{12}}\left(-s_{12}-s_{23}\right)^{d-4}\left(\frac{s_{12}+s_{13}}{s_{12}}\right)^{\frac{d}{2}-3} \\
& { }_{2} F_{1}\left(3-\frac{d}{2}, 4-d ; 5-d ; \frac{s_{13} s_{23}}{\left(s_{12}+s_{13}\right)\left(s_{12}+s_{23}\right)}\right) \\
& -\frac{(3 d-8)(3 d-10)}{4(d-3)(d-4)} A_{3} \frac{1}{s_{12} s_{123}}\left(-s_{13}\right)^{d-3}\left(\frac{s_{12}+s_{23}}{s_{123}}\right)^{\frac{d}{2}-2}\left(\frac{s_{12}+s_{13}}{s_{12}}\right)^{\frac{d}{2}-3} \\
& { }_{2} F_{1}\left(\frac{d}{2}-1,1 ; d-2 ; \frac{s_{13}}{s_{123}}\right){ }_{2} F_{1}\left(3-\frac{d}{2}, 4-d ; 5-d ; \frac{s_{13} s_{23}}{\left(s_{12}+s_{13}\right)\left(s_{12}+s_{23}\right)}\right) \\
& -\frac{(3 d-8)(3 d-10)}{2(d-4)^{2}} A_{3} \frac{1}{-s_{12}-s_{23}}\left(-s_{13}\right)^{d-4}\left(\frac{s_{12}+s_{23}}{s_{12}+s_{13}}\right)^{\frac{d}{2}-1}\left(\frac{s_{123}}{s_{12}}\right)^{\frac{d}{2}-2} \\
& F_{1}\left(4-d, 2-\frac{d}{2}, 2-\frac{d}{2}, 5-d, \frac{s_{23}}{s_{12}+s_{23}}, \frac{s_{23}}{s_{123}}\right) \text {, }
\end{aligned}
$$

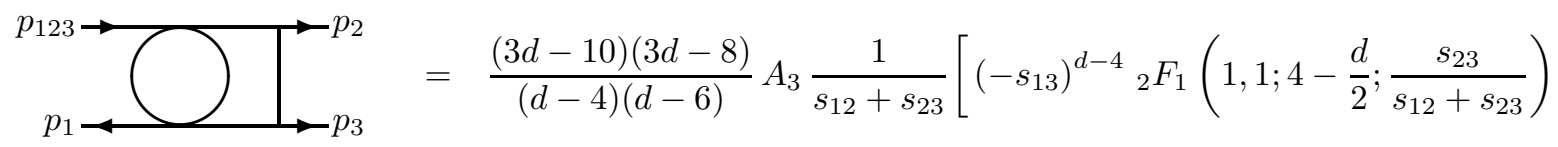

$$
\begin{aligned}
& \left.-\left(-s_{123}\right)^{d-4} F_{1}\left(1,1,4-d, 4-\frac{d}{2} ; \frac{s_{23}}{s_{12}+s_{23}}, \frac{s_{23}}{s_{123}}\right)\right] \\
& -\frac{2(3 d-8)(3 d-10)}{(d-4)(d-6)} A_{3}\left(-s_{123}\right)^{d-5}{ }_{2} F_{1}\left(1,2-\frac{d}{2} ; \frac{d}{2}-1 ; \frac{s_{12}}{s_{12}+s_{13}}\right) \\
& {\left[{ }_{2} F_{1}\left(1,5-d ; 4-\frac{d}{2} ; \frac{s_{23}}{s_{123}}\right)+\frac{d-6}{d-4}{ }_{2} F_{1}\left(1,5-d ; 3-\frac{d}{2} ; \frac{s_{12}+s_{13}}{s_{123}}\right)\right],(5 \text {. }} \\
& p_{1 \rightarrow D_{-p_{3}}} \mathrm{D}_{123 \rightarrow p_{2}}^{p_{2}} A_{3} \frac{1}{s_{13} s_{23}}[ \\
& -\left(\frac{s_{13} s_{23}}{-\left(s_{12}+s_{23}\right)}\right)^{d-3}{ }_{2} F_{1}\left(d-3, d-3 ; d-2 ; \frac{s_{12}}{s_{12}+s_{23}}\right) \\
& -\left(\frac{s_{13} s_{23}}{-\left(s_{12}+s_{13}\right)}\right)^{d-3}{ }_{2} F_{1}\left(d-3, d-3 ; d-2 ; \frac{s_{12}}{s_{12}+s_{13}}\right) \\
& \left.+\left(\frac{-s_{123} s_{13} s_{23}}{\left(s_{12}+s_{23}\right)\left(s_{12}+s_{13}\right)}\right)^{d-3}{ }_{2} F_{1}\left(d-3, d-3 ; d-2 ; \frac{s_{12} s_{123}}{\left(s_{12}+s_{13}\right)\left(s_{12}+s_{23}\right)}\right)\right] \\
& p_{1 \rightarrow p^{p_{123} \rightarrow p_{3}}} p^{p_{2}}=-A_{4} \frac{(d-3)(3 d-10)}{(d-4)^{2}}\left(-s_{123}\right)^{d-4}\left(-s_{13}-s_{23}\right)^{3-d} \\
& {\left[\left(-s_{23}\right)^{d-4} F_{1}\left(4-d, d-3,2-\frac{d}{2}, 5-d, \frac{s_{13}}{s_{13}+s_{23}}, \frac{s_{13}}{s_{123}}\right)\right.} \\
& \left.+\left(-s_{13}\right)^{d-4} F_{1}\left(4-d, d-3,2-\frac{d}{2}, 5-d, \frac{s_{23}}{s_{13}+s_{23}}, \frac{s_{23}}{s_{123}}\right)\right]
\end{aligned}
$$




$$
\begin{aligned}
& -A_{3} \frac{(3 d-8)(3 d-10)}{2(d-4)^{2}} \frac{1}{-s_{123}} \\
& {\left[\left(-s_{13}\right)^{d-4} S_{1}\left(\frac{d}{2}-1,1,1, d-2,5-d, \frac{s_{23}}{s_{123}}, \frac{s_{13}}{s_{123}}\right)\right.} \\
& \left.+\left(-s_{23}\right)^{d-4} S_{1}\left(\frac{d}{2}-1,1,1, d-2,5-d, \frac{s_{13}}{s_{123}}, \frac{s_{23}}{s_{123}}\right)\right] .
\end{aligned}
$$

The integrals (5.25) and (5.26) are one-loop bubble insertions into the one-loop box and have already been computed for arbitrary powers of the propagators in [10]. The integrals (5.27) and (5.28) were, to our knowledge, not known up to now. In the reduction of integrals of the topology (5.28), one finds two master integrals, whose differential equations decouple in the variable $\Delta \equiv s_{13}-s_{23}$. The second master integral for this topology can be found by rearranging one of the differential equations:

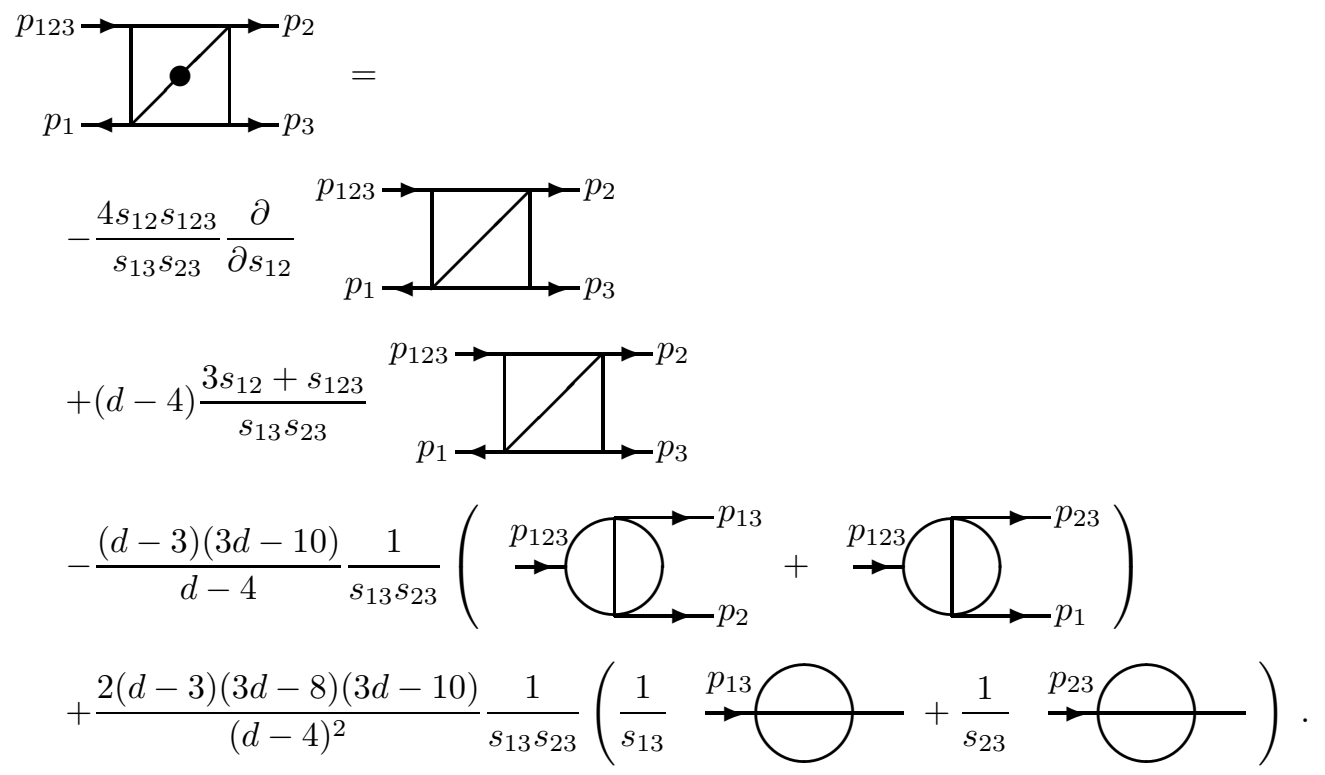

Finally, products of one-loop vertex with one-loop bubble integrals also yield topologies with $t=5$. These can all be reduced to (5.5) and are not quoted explicitly.

The complete list of integrals at $t=5$ which were derived in this section can now be used to compute all integrals at $t=6$ and $t=7$ which can be reduced using IBP and LI identities. The results of this reduction are summarised in the following.

\section{$5.4 t=6$}

Two-loop integrals with $t=6$ arising in calculations in covariant gauges must be three- or four-point functions. Since we are concerned with subgraphs that can appear in the reduction of four-point functions with one off-shell leg, we need to consider three-point functions with up to two off-shell legs. For general three-point functions at $t=6$, one finds three distinct topologies: two planar and one crossed arrangement of the loop momenta. The crossed graphs correspond to master integrals, while the planar graphs are reducible, as first pointed out in [15], where the three-point integral with one off-shell leg was computed. We reproduce these results:

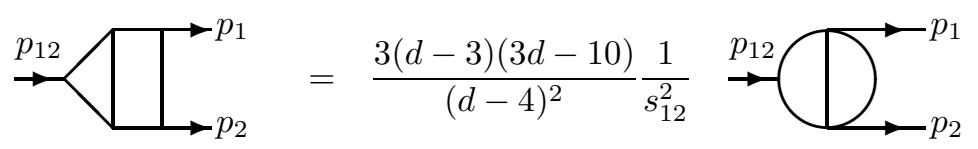




$$
\text { 促 }
$$

The results for two off-shell legs read:

$$
\begin{aligned}
& \rightarrow p_{23}= \\
& s_{123}
\end{aligned}
$$

For $t=6$, both one-loop bubble insertions on propagators of the one-loop box can be reduced:

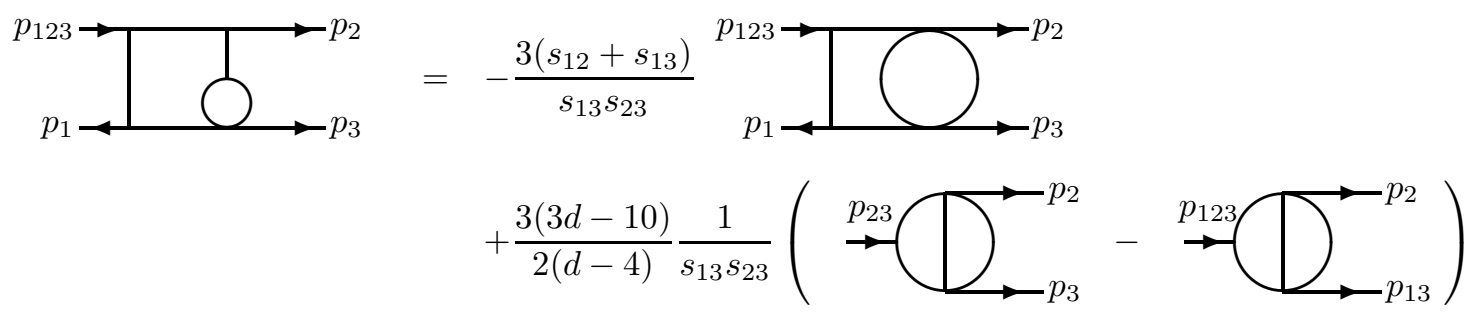




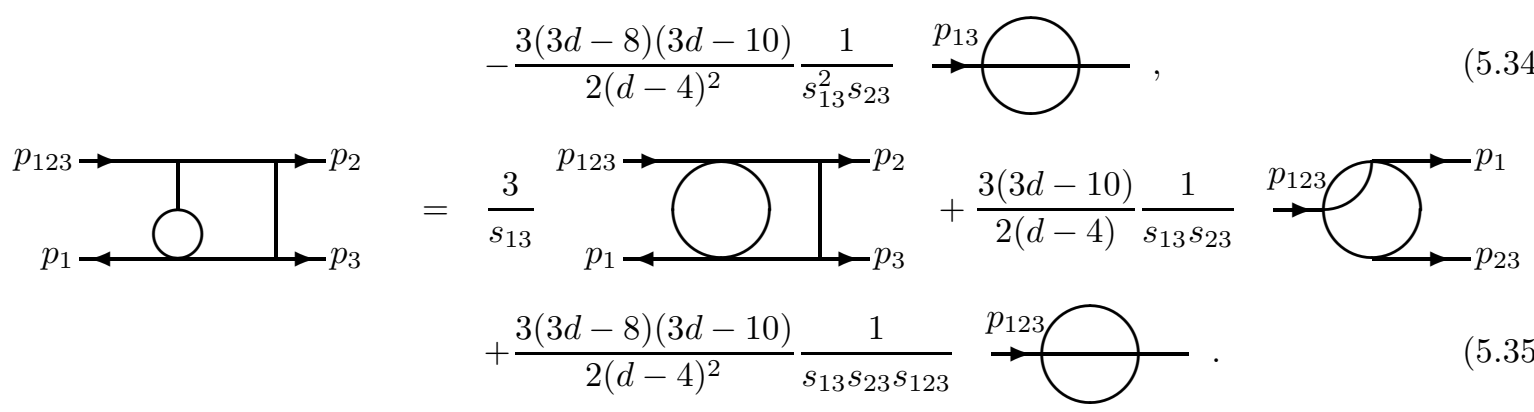

Among the remaining four planar diagrams at $t=6$, three are reducible to simpler subtopologies:

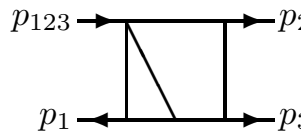

$$
\begin{aligned}
& =-3 \frac{s_{12}}{s_{13} s_{23}} \\
& -\frac{3(d-3)(3 d-10)}{(d-4)^{2}} \frac{1}{s_{13} s_{23}}
\end{aligned}
$$
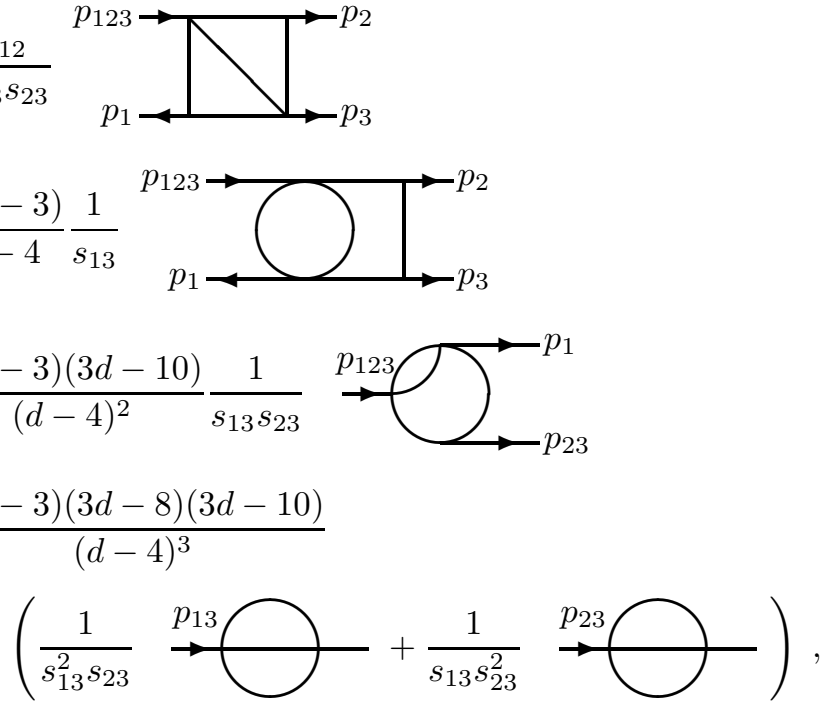
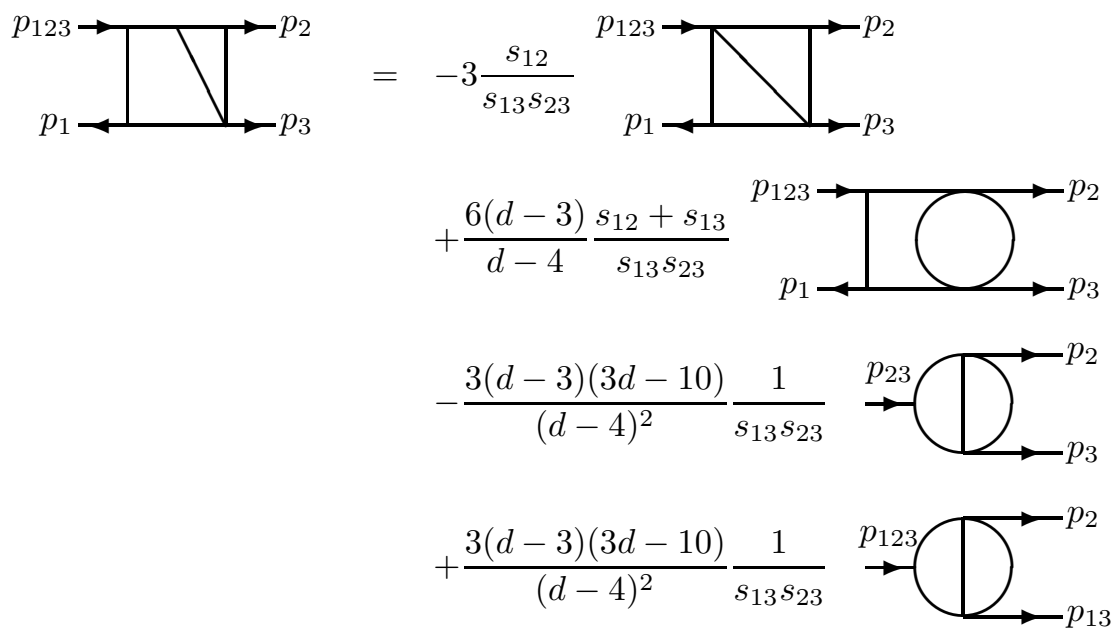

$$
-\frac{3(d-3)(3 d-8)(3 d-10)}{(d-4)^{3}}
$$
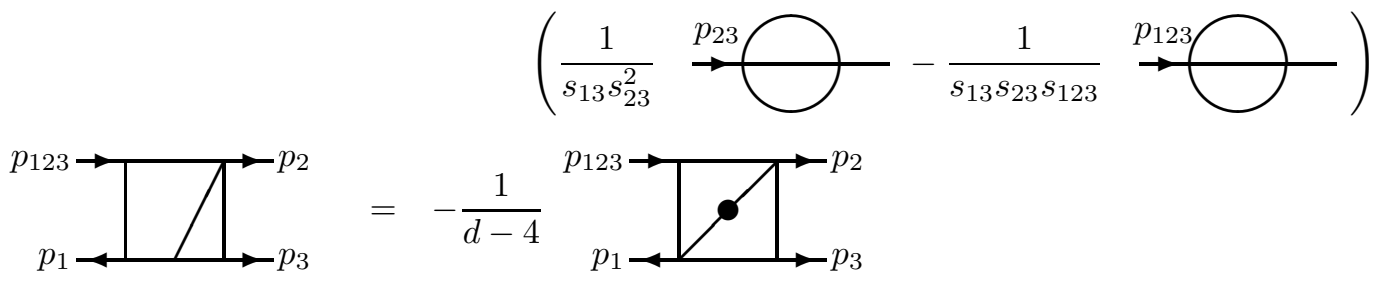

$$
=-\frac{1}{d-4}
$$

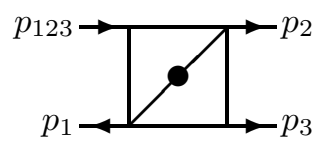




$$
\begin{aligned}
& +\frac{6(d-3)}{d-4} \frac{s_{12}+s_{13}}{s_{13} s_{23}} \quad \begin{array}{c}
p_{123} \rightarrow \\
p_{1} \longrightarrow{ }^{\longrightarrow} p_{3}
\end{array} \\
& -\frac{3(d-3)(3 d-10)}{(d-4)^{2}} \frac{1}{s_{13} s_{23}} \stackrel{p_{23}}{\longrightarrow} \coprod^{-p_{2}} \\
& +\frac{3(d-3)(3 d-10)}{(d-4)^{2}} \frac{1}{s_{13} s_{23}} \stackrel{p_{123}}{\longrightarrow} \coprod^{p_{2}} \\
& +\frac{3(d-3)(3 d-8)(3 d-10)}{(d-4)^{3}} \frac{1}{s_{13}^{2} s_{23}} \stackrel{p_{13}}{\longrightarrow} \square
\end{aligned}
$$

One of the two remaining non-planar diagrams is also reducible, the other non-planar topology contains two master integrals. The reducible integral reads:

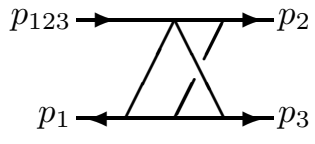

$$
\begin{gathered}
=\frac{3(d-4)}{2 d-9} \frac{s_{12}}{s_{13} s_{23}} \\
+\frac{3(d-4)}{2 d-9} \frac{s_{23}}{s_{12} s_{13}} \\
+\frac{3(d-4)}{2 d-9} \frac{s_{13}}{s_{12} s_{23}} \\
+\frac{3(d-3)(3 d-8)(3 d-10)}{(d-4)^{2}(2 d-9)} \frac{s_{13}+s_{23}}{s_{12}^{2} s_{13} s_{23}} \\
+\frac{3(d-3)(3 d-8)(3 d-10)}{(d-4)^{2}(2 d-9)} \frac{s_{12}+s_{23}}{s_{12} s_{13}^{2} s_{23}} \\
+\frac{3(d-3)(3 d-8)(3 d-10)}{(d-4)^{2}(2 d-9)} \frac{p_{12}+s_{13}}{s_{12} s_{13} s_{23}^{2}}
\end{gathered}
$$

\section{$5.5 \quad t=7$}

At $t=7$ different one finds six different topologies. Three of them are triangle insertions to the one-loop box. These three integrals are all reducible, two of them contain only master integrals up to $t=5$ in their reduction:

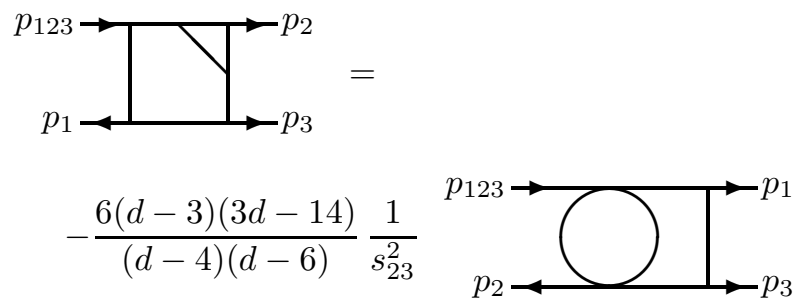




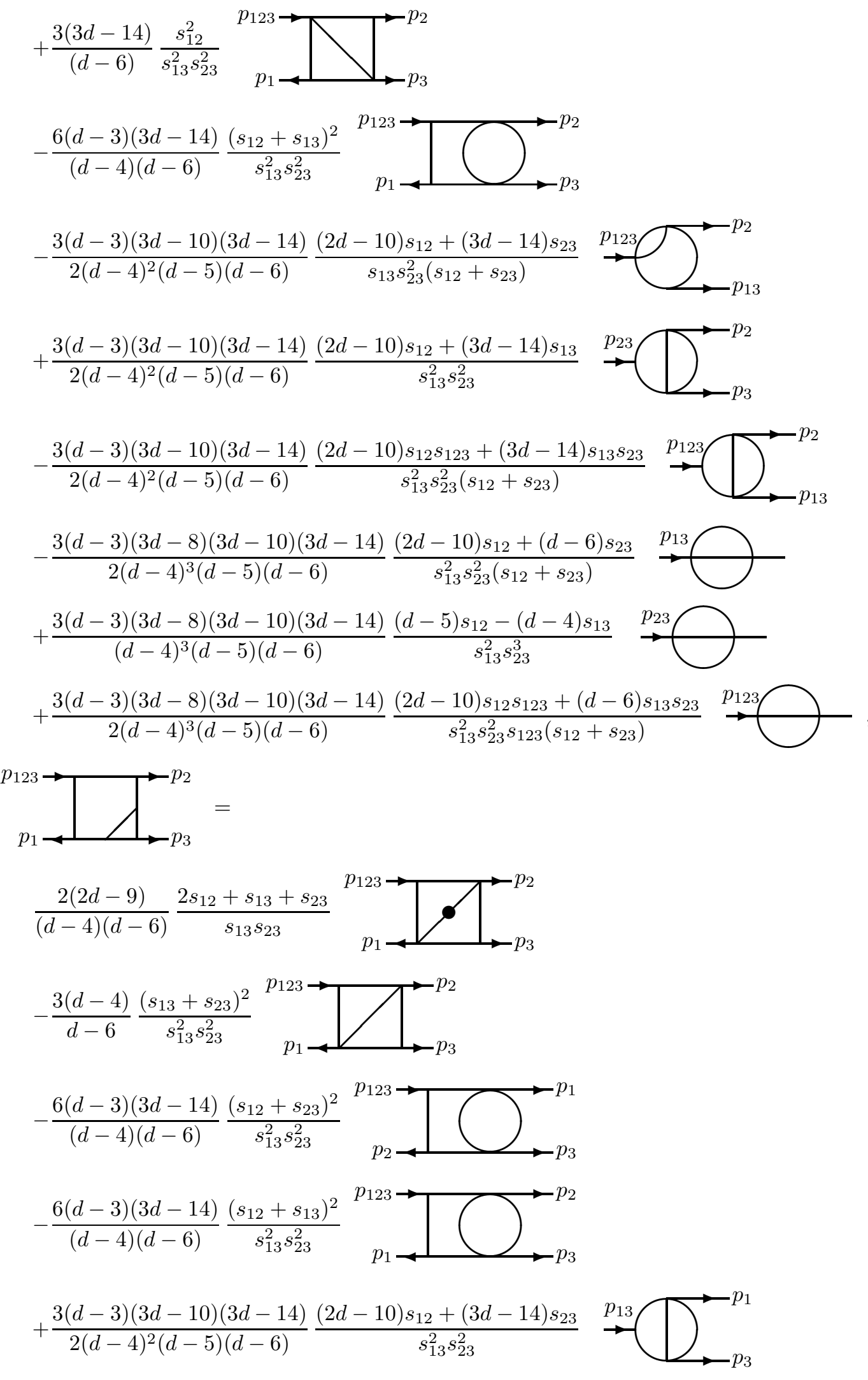




$$
\begin{aligned}
& +\frac{3(d-3)(3 d-10)(3 d-14)}{2(d-4)^{2}(d-5)(d-6)} \frac{(2 d-10) s_{12}+(3 d-14) s_{13}}{s_{13}^{2} s_{23}^{2}} \\
& -\frac{3(d-3)(3 d-10)}{(d-4)^{2}(d-6)} \frac{(3 d-14) s_{12}+(4 d-18) s_{13}-(d-4) s_{23}}{s_{13}^{2} s_{23}^{2}} \\
& -\frac{3(d-3)(3 d-10)}{(d-4)^{2}(d-6)} \frac{(3 d-14) s_{12}-(d-4) s_{13}+(4 d-18) s_{23}}{s_{13}^{2} s_{23}^{2}} \\
& -\frac{3(d-3)(3 d-8)(3 d-10)}{(d-4)^{3}(d-5)(d-6)} \frac{(d-5)(3 d-14)\left(s_{12}+s_{13}\right)+(d-4)^{2} s_{23}}{s_{13}^{3} s_{23}^{2}} \\
& -\frac{3(d-3)(3 d-8)(3 d-10)}{(d-4)^{3}(d-5)(d-6)} \frac{(d-5)(3 d-14)\left(s_{12}+s_{23}\right)+(d-4)^{2} s_{13}}{s_{13}^{2} s_{23}^{3}}
\end{aligned}
$$

The remaining three topologies are the double box and two different momentum arrangements of the crossed box. These topologies contain each two master integrals.

\section{Conclusions and Outlook}

Progress in the computation of exclusive observables, such as for example jet production rates, beyond the next-to-leading order has up to now been hampered mainly by difficulties in the calculation of virtual two-loop integrals with more than two external legs. In contrast to this, many inclusive observables (which correspond from the calculational point of view to two-point functions) are known to next-tonext-to-leading order and even beyond. These higher order calculations relied on a variety of elaborate technical tools for the computation of the virtual integrals. In this paper, we outline how techniques known from multi-loop calculations of two-point integrals can be modified and extended towards the computation of integrals with a larger number of external legs.

We demonstrate that the large number of different two-loop integrals appearing in an actual calculation can be reduced to a small number of scalar master integrals by using the well-known integration-by-parts identities [2, 3] together with identities following from Lorentz-invariance which are unique to multi-leg

integrals. As a by-product of this reduction, one is also able to reduce two-loop integrals with tensorial structure to scalar integrals. In contrast to two-point integrals, where only a few topologically different graphs can appear with potentially large powers of propagators and scalar products, one finds that the reduction of three- and four-point integrals gives rise to a large number of topologically different graphs, which appear however only with small powers of propagators and scalar products. The reduction of twopoint functions usually proceeds via solving manually the integration-by-parts identities for arbitrary powers of propagators and denominators in a given graph topology; this procedure seems to be not practicable for multi-leg integrals. To accomplish the reduction of these, we developed an algebraic program which automatically derives and solves the integration-by-parts and Lorentz-invariance identities for a given graph up to some pre-selected fixed number of powers in denominators and scalar products independent of the topology.

To compute the scalar master integrals, we derive differential equations in the external momenta [13] for them; the boundary conditions of these differential equations correspond to simpler integrals, where for example one of the external momenta vanishes. These differential equations can be solved (for arbitrary space-time dimensions) by employing standard mathematical methods. We observe that the differential equations for the master integrals we considered up to now are solved by generalised hypergeometric functions. We illustrate the application of this method in detail on the example of the one-loop fourpoint function with one off-shell leg.

Using the differential equation method, we provide a complete list of all master integrals with up 
to $t=5$ different denominators that can appear in the reduction of two-loop four-point functions with one off-shell leg. We also list all reducible integrals with $t=6$ and $t=7$ different propagators. The computation of the master integrals with $t=6$ and $t=7$ is still an outstanding task.

The differential equations for these outstanding master integrals are of similar structure as the differential equations for master integrals with a smaller number of different propagators. The main problem towards a complete computation of these integrals is at present the integration of the inhomogeneous term, containing itself already hypergeometric functions arising from the subtopologies.

It is worthwhile to point out similarities and differences between the differential equation method employed in this paper and other methods employed for similar calculations in the literature. Both the negative dimension approach of [10] and the Mellin-Barnes transformation method employed in [8, 9] rely on choosing a particular assignment of momentum vectors to the internal loop propagators. After this assignment, a representation of the propagators in terms of a multiple sum (negative dimension approach) or an integral transformation (Mellin-Barnes method) is employed, such that the integral over the loop momentum can be carried out explicitly. The final result for the integral is then retrieved by resummation of a multiple sum or by an inverse integral transformation. Both methods, when employed for arbitrary space-time dimension, give rise to generalised hypergeometric functions, which can be represented as multiple sums as well as inverse Mellin-Barnes integrals [17]. In the differential equation method, one assigns momentum vectors to the loop propagators only for the sake of deriving the differential equations and the IBP and LI identities. After applying these identities to simplify the differential equations, one obtains a relation between the derivative (with respect to an external momentum) of a master integral, the master integral itself and other master integrals with simpler topology, independent of the parametrisation chosen for the internal propagators. These differential equations can then be solved analytically by integration; the resulting integrals correspond to the integral representations of generalised hypergeometric functions [17]. Using the differential equation method, one can therefore circumvent the explicit loop momentum integration needed in the other methods and one arrives at a representation of the hypergeometric functions, which is presumably more transparent than a multiple sum or an inverse integral transformation. In the integral representation, it is in particular straightforward to identify linear combinations of different hypergeometric functions, which are difficult to disentangle in the other representations. At present, it should however not be claimed that any of the methods is superior, since none of them could yet be employed to compute all outstanding two-loop four-point master integrals.

As a final point, we note that the methods derived in this paper contain a high level of redundancy, which allows for a number of non-trivial checks on the results obtained with them. The automatic reduction to master integrals using integration-by-parts and Lorentz-invariance identities corresponds to the solution of a linear system of equations containing more identities than unknowns. The existence of a solution to this system provides therefore already a check on the self-consistency of the identities. In computing the master integrals from differential equations, one integrates one of the three differential equations in the external invariants, such that the result can be checked by inserting it in the remaining two differential equations.

In short, this paper demonstrates how techniques developed for multi-loop calculation of two-point functions can be extended towards integrals with a larger number of external legs. As a first example of the application of these tools in practice, we computed some up to now unknown two-loop four-point functions, relevant for jet calculus beyond the next-to-leading order. The most important potential application of these tools is the yet outstanding derivation of two-loop virtual corrections to exclusive quantities, such as jet observables.

\section{Acknowledgements}

We are grateful to Jos Vermaseren for his assistance in the use of the algebraic program FORM. One of the authors (E.R.) wants to thank the Alexander-von-Humboldt Stiftung for supporting his stay at the Institut für Theoretische Teilchenphysik of the University of Karlsruhe. The research work presented in this paper was supported in part by the DFG (Forschergruppe "Quantenfeldtheorie, Computeralgebra und Monte-Carlo Simulation", contract KU 502/8-2). 


\section{A Special Functions}

This appendix summarises the series and integral representations of the hypergeometric functions appearing in the master integrals. The properties of these functions, in particular their region of analyticity, their analytic continuation as well as reduction formulae, can be found in the literature [10, 17, 18, 19].

Hypergeometric functions are sums with coefficients formed from Pochhammer symbols

$$
(a)_{n} \equiv \frac{\Gamma(a+n)}{\Gamma(a)} .
$$

The hypergeometric functions of a single variable are given by:

$$
\begin{aligned}
{ }_{2} F_{1}(a, b ; c ; z) & =\sum_{n=0}^{\infty} \frac{(a)_{n}(b)_{n}}{(c)_{n}} \frac{z^{n}}{n !}, \\
{ }_{3} F_{2}\left(a, b_{1}, b_{2} ; c_{1}, c_{2} ; z\right) & =\sum_{n=0}^{\infty} \frac{(a)_{n}\left(b_{1}\right)_{n}\left(b_{2}\right)_{n}}{\left(c_{1}\right)_{n}\left(c_{2}\right)_{n}} \frac{z^{n}}{n !} .
\end{aligned}
$$

Two types of hypergeometric functions of two variables also appear in our results:

$$
\begin{aligned}
F_{1}\left(a, b_{1}, b_{2} ; c ; z_{1}, z_{2}\right) & =\sum_{m, n=0}^{\infty} \frac{(a)_{m+n}\left(b_{1}\right)_{m}\left(b_{2}\right)_{n}}{(c)_{m+n}} \frac{z_{1}^{m}}{m !} \frac{z_{2}^{n}}{n !}, \\
S_{1}\left(a_{1}, a_{2}, b ; c, d ; z_{1}, z_{2}\right) & =\sum_{m, n=0}^{\infty} \frac{\left(a_{1}\right)_{m+n}\left(a_{2}\right)_{m+n}(b)_{m}}{(c)_{m+n}(d)_{m}} \frac{z_{1}^{m}}{m !} \frac{z_{2}^{n}}{n !} .
\end{aligned}
$$

These functions have the following integral representations:

$$
\begin{aligned}
{ }_{2} F_{1}(a, b ; c ; z)= & \frac{\Gamma(c)}{\Gamma(b) \Gamma(c-b)} \int_{0}^{1} \mathrm{~d} t t^{b-1}(1-t)^{c-b-1}(1-t z)^{-a} \\
{ }_{3} F_{2}\left(a, b_{1}, b_{2} ; c_{1}, c_{2} ; z\right)= & \frac{\operatorname{Re}(b)>0, \quad \operatorname{Re}(c-b)>0}{\Gamma\left(b_{1}\right) \Gamma\left(c_{1}-b_{1}\right) \Gamma\left(b_{2}\right) \Gamma\left(c_{2}-b_{2}\right)} \\
& \int_{0}^{1} \mathrm{~d} t_{1} \int_{0}^{1} \mathrm{~d} t_{2} t_{1}^{b_{1}-1} t_{2}^{b_{2}-1}\left(1-t_{1}\right)^{c_{1}-b_{1}-1}\left(1-t_{2}\right)^{c_{2}-b_{2}-1}\left(1-t_{1} t_{2} z\right)^{-a} \\
\operatorname{Re}\left(b_{1}\right)>0, \quad \operatorname{Re}\left(c_{1}-b_{1}\right)>0, \quad \operatorname{Re}\left(b_{2}\right)>0, \quad \operatorname{Re}\left(c_{2}-b_{2}\right)>0 & \frac{\Gamma(c)}{\Gamma(a) \Gamma(c-a)} \int_{0}^{1} \mathrm{~d} t t^{a-1}(1-t)^{c-a-1}\left(1-t z_{1}\right)^{-b_{1}}\left(1-t z_{2}\right)^{-b_{2}} \\
F_{1}\left(a, b_{1}, b_{2} ; c ; z_{1}, z_{2}\right)= & \frac{\operatorname{Re}(a)>0, \quad \operatorname{Re}(c-a)>0}{\Gamma\left(a_{1}\right) \Gamma\left(c-a_{1}\right) \Gamma(b) \Gamma(d-b)} \\
S_{1}\left(a_{1}, a_{2}, b ; c, d ; z_{1}, z_{2}\right)= & \int_{0}^{1} \mathrm{~d} t_{1} \int_{0}^{1} \mathrm{~d} t_{2} t_{1}^{a_{1}-1} t_{2}^{b-1}\left(1-t_{1}\right)^{c-a_{1}-1}\left(1-t_{2}\right)^{d-b-1}\left(1-t_{1} t_{2} z_{1}-t_{1} z_{2}\right)^{-a_{2}} \\
& \operatorname{Re}\left(a_{1}\right)>0, \quad \operatorname{Re}\left(c-a_{1}\right)>0, \quad \operatorname{Re}(b)>0, \quad \operatorname{Re}(d-b)>0
\end{aligned}
$$

\section{B Expansion of Hypergeometric Functions}

To separate divergent and finite parts of the loop integrals derived in this paper, one has to expand them around the physical number of space-time dimensions in the parameter $\epsilon=(4-d) / 2$. We demonstrate 
in this appendix, that this expansion can, at least for the hypergeometric functions in one variable, be carried out in a mechanical way, giving rise to harmonic polylogarithms (HPL), a generalisation of Nielsen's polylogarithms 23] introduced in [24.

Expanding the integral representation of ${ }_{2} F_{1}$ in $\epsilon$ yields simple powers of $(t, 1-t, 1-t z)$ times the product of some number of $(\ln t, \ln (1-t), \ln (1-t z))$. The powers of $(t, 1-t, 1-t z)$ can be integrated by parts until one obtains non-trivial integrals

$$
\int_{0}^{1} \mathrm{~d} t\left(\frac{1}{t}, \frac{1}{1-t}, \frac{1}{1-t z}\right) \ln ^{n_{1}} t \ln ^{n_{2}}(1-t) \ln ^{n_{3}}(1-t z) .
$$

All these integrals are combinations of harmonic polylogarithms $H(\vec{a} ; z)$, where $\vec{a}$ is a vector of indices with $w=n_{1}+n_{2}+n_{3}+1$ components. $w$ is called the weight of the harmonic polylogartihm. The proof by induction in $w$ is trivial, once the HPL formalism [24 is recalled:

1. Definition of the three HPLs at $w=1$ :

$$
\begin{aligned}
H(1 ; z) & \equiv-\ln (1-z) \\
H(0 ; z) & \equiv \ln z \\
H(-1 ; z) & \equiv \ln (1+z)
\end{aligned}
$$

and the three fractions

$$
\begin{aligned}
f(1 ; z) & \equiv \frac{1}{1-z}, \\
f(0 ; z) & \equiv \frac{1}{z}, \\
f(-1 ; z) & \equiv \frac{1}{1+z},
\end{aligned}
$$

such that

$$
\frac{\partial}{\partial z} H(a ; z)=f(a ; z) \quad \text { with } \quad a=+1,0,-1 .
$$

2. For $w>1$ :

$$
\begin{aligned}
H(0, \ldots, 0 ; z) & \equiv \frac{1}{w !} \ln ^{w} z \\
H(a, \vec{b} ; z) & \equiv \int_{0}^{z} \mathrm{~d} x f(a ; x) H(\vec{b} ; x)
\end{aligned}
$$

which results in

$$
\frac{\partial}{\partial z} H(a, \vec{b} ; z)=f(a ; z) H(\vec{b} ; z)
$$

This last relation is a convenient tool for verifying identities among different HPLs. Such identities can be verified by first checking a special point (typically $z=0$ ) and subsequently checking the derivatives. If agreement in the derivatives is not obvious, this procedure can be repeated until one arrives at relations involving only HPLs with $w=1$.

3. The HPLs fulfil an algebra (see Section 3 of [24]), such that a product of two HPLs (with weights $w_{1}$ and $w_{2}$ ) of the same argument $z$ is a combination of HPLs of argument $z$ with weight $w=w_{1}+w_{2}$.

Using these properties of the HPL, one can show that the integrals appearing in the $\epsilon$-expansion of the hypergeometric function can be reexpressed as

$$
\int_{0}^{1} \mathrm{~d} t\left(\frac{1}{t}, \frac{1}{1-t}, \frac{1}{1-t z}\right) \ln ^{n_{1}} t \ln ^{n_{2}}(1-t) \ln ^{n_{3}}(1-t z) \rightarrow \int_{0}^{1} \mathrm{~d} t\left(\frac{1}{t}, \frac{1}{1-t}, \frac{1}{t-1 / z}\right) H(\vec{a}, t) H(\vec{b}, z t) .
$$


Following the argumentation of Section 7 of [24], one can show that the integral on the right hand side of the above equation yields a linear combination of HPLs of weight $w=w_{a}+w_{b}+1$. The proof goes via induction in $w_{b}$.

For $w_{a}=w_{b}=0$ one has $H(\vec{a} ; t) H(\vec{b} ; z t)=1$. The $t$-integral in (B.7) yields then a combination of HPL of weight $w=1$ (B.1). Likewise, for $w_{b}=0$ the right hand side of (B.7) will yield a linear combination of HPLs of weight $w=w_{a}+1$ and of argument $z$, as proven in Section 7 of [24].

Considering

$$
\frac{\partial}{\partial z} \int_{0}^{1} \mathrm{~d} t\left(\frac{1}{t}, \frac{1}{1-t}, \frac{1}{t-1 / z}\right) H(\vec{a} ; t) H(B, \vec{b} ; z t)
$$

we observe that

$$
\begin{aligned}
\frac{\partial}{\partial z} \frac{\mathrm{d} t}{t-1 / z} & =\frac{1}{z^{2}} \frac{1}{t-1 / z} \mathrm{~d} t \frac{\partial}{\partial t}+\text { boundary terms } \\
\frac{\partial}{\partial z} H(B, \vec{b} ; z t) & =t f(B ; z t) H(\vec{b} ; z t) .
\end{aligned}
$$

Making these replacements in (B.8) and applying partial fractioning to all denominators, we are left with

$$
\left(\frac{1}{z}, \frac{1}{1-z}, \frac{1}{1+z}\right) \int_{0}^{1} \mathrm{~d} t\left(\frac{1}{t}, \frac{1}{1-t}, \frac{1}{t-1 / z}\right) H(\vec{a} ; t) H(\vec{b} ; z t)
$$

which is a combination of HPLs with argument $z$ and weight $w=w_{a}+w_{b}+1$ multiplied with $(1 / z, 1 /(1-$ $z), 1 /(1+z)$ ). Integrating (B.8) over $z$ will thus yield a combination of HPLs with argument $z$ and weight $w+1$, which completes the proof by induction.

The $\epsilon$-expansion of ${ }_{3} F_{2}$, corresponding to a double integral in $t_{1}$ and $t_{2}$, is obtained by carrying out the procedure described here twice, again resulting in a combination of HPLs. A systematic $\epsilon$-expansion of $F_{1}$ and $S_{1}$, which are functions of two variables $z_{1}$ and $z_{2}$, will in general go beyond the harmonic polylogarithms in one variable.

\section{References}

[1] C.G. Bollini and J.J. Giambiagi, Nuovo Cim. 12B (1972) 20;

G.M. Cicuta and E. Montaldi, Nuovo Cim. Lett. 4 (1972) 329.

[2] G. 't Hooft and M. Veltman, Nucl. Phys. B44 (1972) 189.

[3] F.V. Tkachov, Phys. Lett. 100B (1981) 65;

K.G. Chetyrkin and F.V. Tkachov, Nucl. Phys. B192 (1981) 159.

[4] K.G. Chetyrkin, J.H. Kühn and A. Kwiatkowski, Phys. Rept. 277 (1996) 189.

[5] S. Laporta and E. Remiddi, Phys. Lett. B379 (1996) 283 (hep-ph/9602417).

[6] J.A.M. Vermaseren, Symbolic Manipulation with FORM, Version 2, CAN, Amsterdam, 1991.

[7] MAPLE V Release 3, Copyright 1981-1994 by Waterloo Maple Software and the University of Waterloo.

[8] V.A. Smirnov, Phys. Lett. B460 (1999) 397 (hep-ph/9905323).

[9] J.B. Tausk, Phys. Lett. B469 (1999) 225 (hep-ph/9909506).

[10] C. Anastasiou, E.W.N. Glover and C. Oleari, Nucl. Phys. B565 (2000) 445 (hep-ph/9907523); Nucl. Phys. B572 (2000) 307 (hep-ph/9907494).

[11] V.A. Smirnov and O.L. Veretin, Nucl. Phys. B566 (2000) 469 (hep-ph/9907385). 
[12] A.V. Kotikov, Phys. Lett. B254 (1991) 158.

[13] E. Remiddi, Nuovo Cim. 110A (1997) 1435 (hep-th/9711188);

M. Caffo, H. Czyz, S. Laporta and E. Remiddi, Proceedings of Zeuthen Workshop on Loops and Legs in Gauge Theories, Rheinsberg, 1998, Acta Phys. Polonica 29 (1998) 2627 (hep-th/9807119).

[14] M. Caffo, H. Czyz and E. Remiddi, Nuovo Cim. 111A (1998) 365 (hep-th/9805118).

[15] G. Kramer and B. Lampe, J. Math. Phys. 28 (1987) 945.

[16] E. Kamke, Differentialgleichungen: Lösungsmethoden und Lösungen, Vol. 1 \& 2, B.G. Teubner (Stuttgart, 1977).

[17] A. Erdelyi, W. Magnus, F. Oberhettinger and F. Tricomi (Bateman Manuscript Project), Higher Transcendental Functions, Vol. I, McGraw Hill (New York, 1953).

[18] I.S. Gradshteyn and I.M. Ryzhik, Table of Integrals, Series and Products, Academic Press (Boston, 1980).

[19] H. Exton, Multiple Hypergeometric Functions, Ellis Horwood (Chichester, 1976).

[20] I.N. Bronstein and K.A. Semendjajew, Taschenbuch der Mathematik, BSB B.G. Teubner Verlagsgesellschaft (Leipzig, 1979).

[21] R.K. Ellis, D.A. Ross and A.E. Terrano, Nucl. Phys. B178 (1981) 421.

[22] S. Catani, Phys. Lett. B427 (1998) 161.

[23] N. Nielsen, Nova Acta Leopoldiana (Halle) 90 (1909) 123;

L. Lewin, Polylogarithms and Associated Functions, North Holland (Amsterdam, 1981).

[24] E. Remiddi and J.A.M. Vermaseren, Int. J. Mod. Phys. A15 (2000) 725 (hep-ph/9905237). 